\title{
Upper bounds on the length of a shortest closed geodesic and quantitative Hurewicz theorem
}

\author{
Received November 8, 2001 / final version received March 10, 2003
}

Published online June 6, 2003 - (C) Springer-Verlag \& EMS 2003

\begin{abstract}
In this paper we present two upper bounds on the length of a shortest closed geodesic on compact Riemannian manifolds. The first upper bound depends on an upper bound on sectional curvature and an upper bound on the volume of the manifold. The second upper bound will be given in terms of a lower bound on sectional curvature, an upper bound on the diameter and a lower bound on the volume.

The related questions that will also be studied are the following: given a contractible $k$-dimensional sphere in $M^{n}$, how "fast" can this sphere be contracted to a point, if $\pi_{i}\left(M^{n}\right)=\{0\}$ for $1 \leq i<k$. That is, what is the maximal length of the trajectory described by a point of a sphere under an "optimal" homotopy? Also, what is the "size" of the smallest non-contractible $k$-dimensional sphere in a $(k-1)$-connected manifold $M^{n}$ providing that $M^{n}$ is not $k$-connected?
\end{abstract}

\section{Introduction}

The first objective of this paper is to find explicit upper bounds for the length of a shortest closed geodesic. The first results of this nature were obtained by Loewner and $\mathrm{Pu}$ for 2-dimensional torus and 2-dimensional projective plane, respectively. The upper bound problem has received considerable attention from M. Berger and M. Gromov, (see [Br], [G2]) and was also stated by S.T. Yau as problem 87 in the problem list [Y].)

Among the obtained results giving an upper bound for the length of a shortest closed geodesic on nonsimply-connected manifolds we can mention the fundamental result of M. Gromov, that states that $l(M) \leq c(n) \operatorname{vol}(M)^{\frac{1}{n}}$, where $l(M)$ denotes the length of a shortest closed geodesic, $n$ is the dimension of $M$ providing that $M$ is essential. Recall that $M$ is called essential if there exists a continuous map $f: M \longrightarrow K(\Pi, 1)$ of $M$ into an aspherical space $K(\Pi, 1)$ such that $f_{*}([M]) \neq 0$, where $[M]$ is the fundamental homology class of $M$ and $f_{*}$ is the homomorphism between the homology groups induced by $f$ (see [G2]).

For simply connected manifolds there are results by Ballmann, Thorbergsson and Ziller for nonnegatively curved manifolds (in particular, for spheres endowed

A. Nabutovsky: Department of Mathematics, University of Toronto, Toronto, Ontario M5S 3G3, Canada, e-mail: alex@math.toronto.edu

R. Rotman: Department of Mathematics, 100 St. George, University of Toronto, Toronto, Ontario M5S 3G3, Canada, e-mail: rina@math.toronto.edu 
with a $\frac{1}{4}$-pinched metric of positive sectional curvature), the results of Croke and of Treibergs for convex hypersurfaces, and curvature free result of Croke for the manifold diffeomorphic to the 2-dimensional sphere, (which was somewhat improved by Maeda in [M]) (see [BalThZ], [Cr2], [T]).

The only known upper bounds valid for simply connected Riemannian manifolds of dimension $\geq 3$ with an arbitrary Riemannian metric were obtained in [R]. However, those bounds were obtained only for manifolds with $H_{2}(M) \neq 0$. One of the main goal of this paper is to get rid of this restriction and to find upper bounds valid for all compact Riemannian manifolds.

In this paper we will prove the following two theorems.

Theorem 0.1. Let $M^{n}$ be a compact Riemannian manifold of sectional curvature $K \leq 1$ and volume bounded from above by $V$. Then the length of a shortest closed geodesic $l\left(M^{n}\right) \leq 2 \pi(V+1)^{c(n) V^{n}}$. The same upper bound will hold if the assumption that $K \leq 1$ is replaced by the assumption that the injectivity radius of $M^{n}$ is greater than or equal to $\pi$.

Theorem 0.2. Let $M^{n}$ be a compact Riemannian manifold of sectional curvature $K \geq-1$, diameter $\leq d$ and volume $\geq v$. Then the length of a shortest closed geodesic $l\left(M^{n}\right) \leq \exp \left(\frac{\exp \left(c_{1}(n) d\right)}{\min \{1, v\}^{c_{2}(n)}}\right)$.

In the statement of Theorems $0.1,0.2 c(n), c_{1}(n), c_{2}(n)$ are constants that depend on the dimension $n$ of the manifold only. Our proofs of Theorems 0.1 and 0.2 can be used to obtain some explicit values of these constants, if desired.

It is obvious that Theorems $0.1,0.2$ immediately imply bounds for $l\left(M^{n}\right)$ in the situation when $K$ is not necessarily bounded from above (or below) by 1 since we always can do the appropriate rescaling. So, if $K \leq k^{2}, k \geq 1$ in Theorem 0.1 , then $l\left(M^{n}\right) \leq \frac{2 \pi}{k}\left(V k^{n}+1\right)^{c(n) V^{n} k^{n^{2}}}$. If we assume that $K \geq-k^{2}, k \geq 1$ in Theorem 0.2, then $l\left(M^{n}\right) \leq \frac{1}{k} \exp \left(\frac{\exp \left(c_{1}(n) d k\right)}{\min \left\{1, v k^{n}\right\}^{c_{2}(n)}}\right)$.

In the future, the class of manifolds of dimension $n$, of sectional curvature $K \geq-1$, diameter $\leq d$ and of volume $\geq v$ will be denoted as $\mathcal{M}_{-1, v}^{d}$.

One should note that a lower bound for $l\left(M^{n}\right)$, where $M^{n} \in \mathcal{M}_{-1, v}^{d}$ was discovered by J. Cheeger, (see $[\mathrm{Cg}]$ ).

Before we will discuss the proofs of Theorems 0.1 and 0.2 in any detail, we would like to give a very short outline of it. Theorems 0.1 and 0.2 will be proved as follows. First, one assumes that there is no closed geodesics of length smaller than some fixed $L$. Therefore all closed curves of length less than or equal to $L$ can be contracted without length increase. Next one constructs a non-trivial sphere in the space of closed curves on $M^{n}$ of length less than or equal to $L$ that cannot be "pulled down" to $M^{n}$ along the integral curves of the energy functional $E$ and, thus, obtain a closed geodesic of length less than or equal to $L$ that lies below that sphere as a critical point of $E$.

Now, we note that the construction of a sphere in the space of closed curves on $M^{n}$ is equivalent to the construction of the sphere on $M^{n}$ of one dimension higher. If we want the sphere in the space of closed curves to pass through curves of small 
length then the corresponding sphere on a manifold should have a controllable shape, (thus, we will construct short-tentacled spheres, see Definition 1.2).

In order to construct a sphere of this special shape we will need to learn how to contract contractible spheres "optimally", which in our case means that each point does not travel for too long until it gets to its destination. This is done by induction on the dimension of spheres. The first step of this was essentially done in $[R]$, and uses the assumption about the closed curves being contractible with no length increase.

The rest is done by looking at the proof of the Hurewicz theorem and making it effective. For example, in order to contract a simplicial sphere of dimension $k>1$ we find a chain that it bounds and use it to construct a homotopy that connects this sphere to a point. The details of this procedure will follow.

The starting point of our proof is the Existence theorem of Lusternik and Fet (Theorem 14 in $[\mathrm{F}]$ ) that asserts that on any closed Riemannian manifold there exists at least one closed geodesic. The simplest proof of this theorem (that apparently first appeared in [O]) uses Morse theory on the space of all continuous maps $\alpha: S^{1} \longrightarrow M^{n}$, that will be denoted by $\Lambda M^{n}$ and, roughly speaking, runs as follows, (see also [B], [Ml] for the complete proof).

First, consider the smallest $q$, such that $\pi_{q}\left(M^{n}\right) \neq\{0\}$. Looking at the homotopy exact sequence of the fibration $\Omega M^{n} \longrightarrow \Lambda M^{n} \longrightarrow M^{n}$ one concludes that $\pi_{q-1}\left(\Lambda M^{n}\right) \neq\{0\}$, (whereas $\left.\pi_{q-1}\left(M^{n}\right)=\{0\}\right)$.

Next, consider the energy functional on $\Lambda M^{n}$ and take a non-trivial sphere, (here, by a sphere one will mean the image of a standard sphere under a continuous map) representing a non-trivial element of $\pi_{q-1}\left(\Lambda M^{n}\right)$ and try to deform it along the integral curves of the gradient flow of the energy functional. It is impossible to deform it into $M^{n}$, since one can then contract it in $M^{n}$ and that would contradict non-triviality of that sphere. Thus, it should "get stuck" on a critical point of the energy functional, which is a closed geodesic, (see Fig. 1).

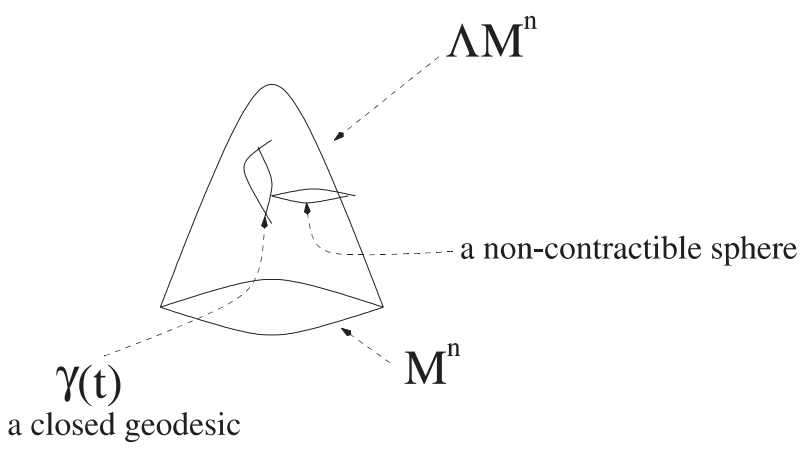

Fig. 1. Illustration of the Theorem of Lusternik and Fet

Let us note that each point of that sphere is a closed curve, and that with respect to the energy functional, or equivalently the length functional, it lies "above" the 
closed geodesic. Therefore, if we could actually construct a non-trivial sphere in $\Lambda M^{n}$, so that the length of curves through which it passes is bounded from above, that would automatically give us an upper bound on the length of a shortest closed geodesic.

As it becomes clear from the proof of the theorem of Lusternik and Fet, our main task is to construct a non-trivial $\tilde{\phi}: S^{q-1} \longrightarrow \Lambda_{L} M^{n}$, where $\Lambda_{L} M^{n}$ is the space of all continuous maps $\alpha: S^{1} \longrightarrow M^{n}$ of length bounded from above by $L$ and where $L$ can be bounded from above in terms of the parameters of the manifold.

This $\tilde{\phi}: S^{q-1} \longrightarrow \Lambda_{L} M^{n}$, can be obtained from $\phi: S^{q} \longrightarrow M^{n}$, "composed" of curves of controlled length, so we can, informally, restate that problem, as the problem of constructing a non-trivial sphere in the manifold that is composed of short curves. (The exact relationship between $\tilde{\phi}$ and $\phi$ will be explained below.)

We will now proceed with an outline of the proof.

\section{(i) The sphere $\phi: S^{q} \longrightarrow M^{n}$ will be obtained as an obstruction to the extension.}

The non-trivial sphere $\phi: S^{q} \longrightarrow M^{n}$ will be obtained as follows: we will start with any non-trivial sphere $\varrho: S^{q} \longrightarrow M^{n}$. The above sphere being non-trivial, the map $\varrho$ cannot be extended to a disc $D^{q+1}$, so our attempts at extending it should fail, and as an obstruction to this extension we will obtain a new non-trivial sphere $\phi: S^{q}=\partial \sigma^{q+1} \longrightarrow M^{n}$, where $\partial \sigma^{q+1}$ is a boundary of a $(q+1)$-dimensional simplex. Since the new sphere will be the result of an explicit construction, we will have a control over its shape and its size.

The new map $\phi$ will be defined on skeleta of $\partial \sigma^{q+1}$ inductively. That is, assuming, we have defined a map on $(q-1)$-skeleton, the map will take $q$-dimensional faces to subsets generated by a homotopy that connects the image of its boundary and a point. We will consider a new sphere to be small if those homotopies are such that the distance travelled by each point under this homotopy is small, i.e. controllable in terms of the parameters of the manifold.

(ii) The sphere $\phi: S^{q} \longrightarrow M^{n}$ can be viewed as $\tilde{\phi}: S^{q-1} \longrightarrow \Lambda_{L} M^{n}$. (For example, consider the identity map $\phi$ of the standard euclidean sphere $S^{n} \subset \mathbf{R}^{n+1}$, and consider its "decomposition" into spheres $F_{t}: S^{n-1} \longrightarrow S^{n}$, such that $F_{t}\left(S^{n-1}\right)=\left\{\sum_{i=1}^{n} x_{i}^{2}=1, x_{n+1}=4 t-1\right\}$, where $t \in\left[0, \frac{1}{2}\right]$ and $F_{t}\left(S^{n-1}\right)=(0, \ldots, 0, \sin 2 \pi t,-\cos 2 \pi t)$, where $t \in[1 / 2,1]$. This can be interpreted as $F:[0,1] \longrightarrow \operatorname{Map}\left(S^{n-1}, S^{n}\right)$, where $F(0)$ and $F(1)$ map $S^{n-1}$ to the south pole of $S^{n}$. Then the length of each closed curve $F_{t}\left(p^{*}\right), t \in[0,1]$ is bounded from above by $2 \pi$, and we can alternatively view this as a map $\tilde{\phi}: S^{n-1} \longrightarrow \Lambda_{2 \pi} S^{n}$.)

A sphere obtained in the way described above can be decomposed into spheres of one dimension smaller as follows: we can construct a map $F:[0,1] \longrightarrow$ $\operatorname{Map}\left(S^{q-1}, M^{n}\right)$, so that $F(t)=F_{t}(p), p \in S^{q-1}$, having the following properties:

(a) $F(0)=F(1)=$ a constant map;

(b) the length of each curve $F(t)\left(p^{*}\right), t \in[0,1], p^{*} \in S^{q-1}$ is fixed, can be bounded from above in terms of the parameters of the manifold. 
In other words, we use here a specific map $\alpha:[0,1] \times S^{q-1} \longrightarrow S^{q}$ which is homotopically just the quotient map from $S^{q-1} \times[0,1]$ to the suspension $\Sigma S^{q-1}=S^{q}$. (This map is explained in more details in Sect. 5, but note that the exact choice of $\alpha$ is not really imortant.) We define $F(t)$ as $(\phi \circ \alpha)(t, *)$.

Or, alternatively, one can view the above map as $\tilde{f}: S^{q-1} \longrightarrow \Lambda_{L} M$, where $\mathrm{L}$ is a $\sup _{p^{*} \in S^{q-1}}$ length of $F_{t}\left(p^{*}\right), t \in[0,1]$, (see Fig. 2), $\tilde{f}(p)=\phi \circ \alpha(*, p)$.

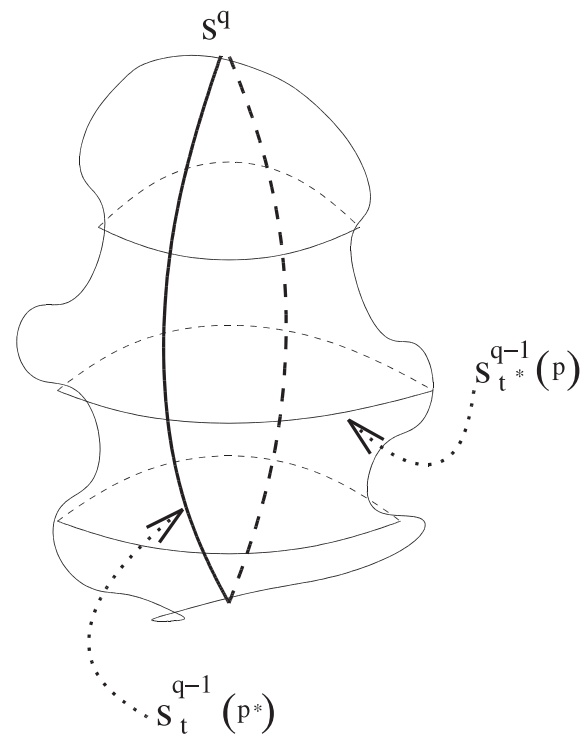

Fig. 2. "Decomposition" of $S^{q}$ into $S^{q-1^{\prime}} S$ reduces the construction of a map $\tilde{\phi}$ from $S^{q-1}$ to $\Lambda M^{n}$ to the construction of a map $\phi: S^{q} \longrightarrow M^{n}$

Steps (i) and (ii) are quite elementary. The crux of the matter lies in step (iii):

(iii) We will use an effective version of the proof of Hurewicz theorem in order to construct homotopies that connect spheres of dimension less than $q$ with a point.

It follows from (i) and (ii) that from the technical point of view our main objective is to be able to construct an "optimal", in a sense of (ii), homotopy for contractible spheres. In order to achieve our goal we will prove results that immediately imply effective version(s) of the Hurewicz theorem. These theorems are the second major goal of the present paper (along with the upper bounds for the length of a shortest contractible geodesic).

The Hurewicz theorem asserts that if $M^{n}$ is $(q-1)$-connected then every $q$-dimensional homology class is representable by a map of $S^{q} \longrightarrow M^{n}$ (the surjectivity of the Hurewicz homomorphism), and that every $q$-dimensional sphere in $M^{n}$ that can be filled by a chain is, in fact, contractible (the injectivity of the Hurewicz homomorphism). This leads to the following natural questions: Let $c$ be a $q$-dimensional chain in $M^{n}$ representing a non-trivial homology class. What is the 
"size" of the "smallest" sphere $S^{q} \longrightarrow M^{n}$ realizing this class? In particular, what is the complexity of the "smallest" sphere realizing a non-trivial $q$-dimensional homology class of $M^{n}$ providing that $H_{q}\left(M^{n}\right) \neq 0$ ? We can regard an answer to these questions as a quantitative version of the surjectivity part of the Hurewicz theorem. On the other hand given the "size" of a $q$-sphere in $M^{n}$ that can be filled by a chain what is the complexity of the "simplest" homotopy contracting the sphere? (An answer to this question can be regarded as a quantitative version of the injectivity of the Hurewicz homomorphism.) In the PL-case it is natural to deal only with simplicial chains and maps and to measure the complexity (or the "size") essentially as the number of simplices they are made of. In the differential-geometric situation we have more possibilities: The most natural measure of complexity of a sphere $f: S^{q} \longrightarrow M^{n}$ or of a homotopy $g$ is the Lipschitz constant Lip $f$ (or Lip g). However, other measures of complexity, such as the width of homotopies can also be of interest. The width of a homotopy is, by definition, the largest distance travelled by a point during the homotopy ([SW]). It is of interest to us because it arises in the proofs of Theorems $0.1,0.2$.

These questions pertain to the field of Quantitative Homotopy Topology. This study of quantitative measures of complexity of maps between Riemannian manifolds the existence of which is predicted by homotopy theory was suggested by M. Gromov (cf. [G 1], [G 3], [G 4]).

The upper bounds that provide the quantitative versions of the Hurewicz theorem use the following information about the geometry of the ambient manifold $M^{n}$. First, we need the number of simplices of $M^{n}$ in the PL-situation (when $M^{n}$ is assumed to be triangulated). In the Riemannian situation we need either:

1. A positive lower bound for the injectivity radius of $M^{n}$ and an upper bound for the volume of $M^{n}$, or

2. A lower bound for the sectional curvature, a positive lower bound for the volume and an upper bound for the diameter.

But that is not enough. We need to know a value of $c\left(M^{n}\right)$ such that any closed curve $\gamma$ on $M^{n}$ can be contracted to a point via curves of length $\leq c\left(M^{n}\right) \times$ the length of $\gamma$. Can one get the desired upper bounds without using $c\left(M^{n}\right)$ ? The answer is both "yes" and "no". "Yes", because there exists a (non-computable) upper bound for $c\left(M^{n}\right)$ in terms of the other available geometric data about $M^{n}$. (The existence of such $c\left(M^{n}\right)$ in the PL-situation immediately follows from the finiteness of the number of triangulations with $\leq N$ simplices. In the Riemannian situation the existence of $c\left(M^{n}\right)$ follows from an easy compactness argument.) "No", because even if $q=2$ (and $n \geq 5$ ) then there is no computable function of the number of simplices of $M^{n}$ that can be used as an upper bound for the minimal size of a non-contractible $q$-sphere. (Here we are talking about the PL-situation. In the Riemannian situation there is no computable function of $|K|, v, D$ that can be used as an upper bound for the minimal size of a non-contractible $q$-sphere, where $|K|$ majorizes the absolute value of the sectional curvature, $D$ majorizes the diameter, and $v>0$ minorizes the volume of $M^{n}$.)

Here are the statements of our results. By a simplicial homotopy contracting a closed simplicial curve $\gamma$ in a triangulated manifold we will mean below a finite 
sequence of closed simplicial curves in $M^{n}$ such that the first curve is $\gamma$, the last curve is a point, and any two consecutive curves can be homotoped to each other through exactly one two-dimensional simplex of $M^{n}$.

Theorem 0.3. Let $M^{n}$ be a closed connected $P L$-manifold triangulated with $\leq N$ simplices. Let $q=\min \left\{i \mid \pi_{i}\left(M^{n}\right) \neq 0\right\}$. Assume that a number $\alpha$ satisfies the following condition: For any $L$ and any closed simplicial curve $\gamma$ in $M^{n}$ made of at most $L$ simplices there exists a simplicial homotopy contracting $\gamma$ to a point passing through simplicial curves made of at most $\alpha$ L simplices. Then there exists a constant $c(n)>0$ depending only on $n$ such that

(a) There exists a triangulation of $S^{q}$ with less than $\exp \left((\alpha N)^{c(n)}\right)$ simplices and a non-contractible simplicial map of this triangulated sphere into $M^{n}$

(b) Any simplicial q-cycle such that the sum of absolute values of its coefficients does not exceed $A$ can be realized by a simplicial map of a q-sphere triangulated with less than $A \exp \left((\alpha N)^{c(n)}\right)$ simplices

(c) Any contractible simplicial map of a triangulated $k$-dimensional sphere $S^{k}$ into $M^{n}$ such that $k \leq q$ and $S^{k}$ is triangulated into $\leq A$ simplices can be extended to a simplicial map of the disc $D^{k+1}$ triangulated into not more than $A \exp \left((\alpha N)^{c(n)}\right)$ simplices.

Theorem 0.4. Let $M^{n}$ be a closed connected Riemannian manifold such that its first $(q-1)$ homotopy groups vanish, but its $q$-th homology group is non-trivial. Assume that for some constant $\alpha$ any closed curve $\gamma$ on $M$ can be contracted to a point via closed curves of length $\leq \alpha \times$ length $(\gamma)$. Assume that either the injectivity radius inj $\left(M^{n}\right) \geq \pi$ or the sectional curvature of $M^{n} \geq-1$. In the first case let $x \geq 2$ denote an upper bound for $\operatorname{vol}\left(M^{n}\right)$, and in the second case let $x \geq 2$ be an upper bound for $\exp \left((n-1) \operatorname{diam}\left(M^{n}\right)\right) / \min \left\{\operatorname{vol}\left(M^{n}\right), 1\right\}$. Then there exists a constant $c(n)$ depending only on $n$ such that

(a) There exists a non-contractible map of $S^{q-1}$ into the space of closed curves $\Lambda M^{n}$ on $M^{n}$ such that its image contains only curves of length $\leq$ $\exp \left((\alpha x)^{c(n)}\right)$

(b) Any map $f: S^{k} \longrightarrow M^{n}$ can be contracted to a point by a homotopy of width not exceeding $\exp \left((\alpha x)^{c(n)}\right)$, providing that $k \leq q-1$ or $k=q$ and $f$ is contractible, (recall, that the width of the homotopy is the maximal distance travelled by a point of the contracted sphere in $M^{n}$ during the homotopy.)

Theorem 0.5. For any $n>4$ there is $N O$ computable function $f_{n}(N)$ with the following property: For any simply connected $n$-dimensional PL-manifold $M^{n}$ triangulated with $\leq N$ simplices and satisfying $H_{2}\left(M^{n}\right) \neq 0$ there exists a triangulation of $S^{2}$ with $\leq f_{n}(N)$ simplices and a non-contractible simplicial map of the resulting simplicial complex into $M^{n}$.

\section{Remarks.}

1. As it was mentioned before the existence of an upper bound $f_{n}(N)$ for the number of simplices in the triangulation of $S^{2}$ required to realize a non-contractible 2-sphere in $M^{n}$ is obvious. It follows from the finiteness of the number of 
$n$-dimensional simplicial complexes with $\leq N$ simplices. The theorem above asserts that such an upper bound cannot be a computable function of $N$.

2. An obvious analog of the theorem holds in the Riemannian geometric situation: There is no computable function of $|K|$, inj, diam, vol, $n, q$ that enables one to majorize the "size" of the "smallest" non-contractible 2-sphere in a closed simply-connected Riemannian manifold.

3. In fact, one can specify a certain PL-homeomorphism type of the manifold $M^{n}$ for any $n \geq 5$ in this theorem.

4. It is not very difficult to prove the existence of (some) upper bounds in Theorems $0.1,0.2$ using an argument based on the precompactness of subsets of the space of isometry classes of all $n$-dimensional Riemannian manifolds of curvature bounded from below and diameter bounded from above (or of the volume bounded from above and the injectivity radius bounded from below). The theorem above helps one to appreciate the constructive (explicit) nature of our upper bounds for the length of a shortest closed geodesic.

Our paper is organized as follows. The next section contains a very detailed description of the scheme of the proof of our main results (Theorems $0.1-0.4$ ). There we also take care of some technical matters that can be easily resolved. In Sect. 2 we introduce the notion of a triangulation tower. Our need for this notion can be very informally explained as follows: Let $M^{n}$ be a Riemannian manifold with injectivity radius $\geq 1$ or contractibility radius $\geq 1$. Consider the nerve of a covering of $M^{n}$ by metric balls of radius $\frac{1}{1000}$. This nerve need not be homotopy equivalent to $M^{n}$ because it can have small size cycles made of several simplices, but its large scale topology will be the same as the topology of $M^{n}$. Consider now the nerve of the covering of $M^{n}$ by balls of radius $\frac{1}{10}$ with the same centres as the balls of the first covering. The first nerve is embedded into the second nerve and all "small" cycles of the first nerve become trivial in the second nerve. However the large scale topology survives. In fact, we need several "nested" nerves of coverings of $M^{n}$ by concentric balls of larger and larger (but still very small!) radii for our purposes. In Sects. 3-5 we explain how to contract spheres using homotopies of bounded width. In Sect. 6 we construct a homotopy non-trivial sphere "made" of free loops of controlled length and as the result establish the upper bounds for the length of a shortest closed geodesic. In Sect. 7 we discuss Theorems 0.3-0.5. In fact, Theorems 0.3, 0.4 follow almost immediately from the results of Sects. 3-6, so the real content of Sect. 7 is the proof of Theorem 0.5. We deduce it from the (well-known) non-existence of an algorithm that decides whether or not a finite presentation of a group is a finite presentation of the trivial group even if it is a priori known that the given finite presentation is a finite presentation of a group $G$ such that its Eilenberg-Maclane space $K(G, 1)$ is homotopy equivalent to a finite $\mathrm{CW}$-complex of dimension at most two.

Finally, note our method yields somewhat more than results asserted by Theorems $0.1,0.2,0.4$. Namely, one can obtain an explicit upper bound for the length of a shortest geodesic only in terms of the following geometric data about $M^{n}$ : 1) A function $N(\varepsilon)$ such that for any $\varepsilon>0$ there exists an $\varepsilon$-net in $M^{n}$ made of not more than $N(\varepsilon)$ points; 2) A number $R>0$ and continuous increas- 
ing functions $\beta, \gamma:[0, R] \longrightarrow[0, \infty], \beta(0)=\gamma(0)=0$, such that any metric ball of radius $x \leq R$ in $M^{n}$ can be contracted to a point inside the concentric ball of radius $\beta(x)$ by a homotopy such that all points travel distances not exceeding $\gamma(x)$. The result of Theorem 0.4 also can be similarly generalized.

\section{The scheme of proof of main results}

1.1. More details on steps (i)-(iii) explained in the introduction. Definitions of the width of a homotopy and short-tentacled spheres. Let us discuss steps (i)-(iii) of the proof of main results outlined in the previous section in some details.

(i) How to obtain a non-trivial sphere $\phi: S^{q} \longrightarrow M^{n}$ ? Let $\varrho: S^{q} \longrightarrow M^{n}$ represent any non-trivial element of $\pi_{q}\left(M^{n}\right)$. We are going to try to extend $\varrho$ to $D^{q+1}$ that fills $S^{q}$. As an obstruction we will obtain a different non-contractible sphere on $M^{n}$.

To demonstrate how it works we will use as an example the case of $q=2$. That is, we want to extend $\varrho: S^{2} \longrightarrow M^{n}$ to $D^{3}$. We will use induction on skeleta of $D^{3}$, (see Fig. 3).
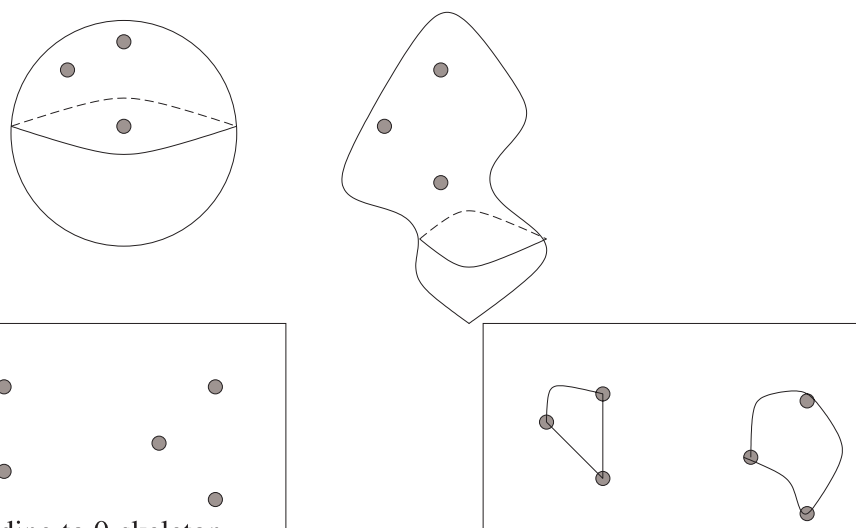

Extending to 1-skeleton

a

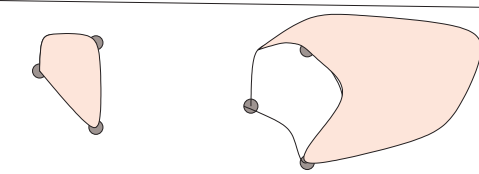

Extending to 2-skeleton

c

Fig. 3. Extending a map $\varrho$ from a sphere to a disc 
We will begin by triangulating $S^{2}$, so that the simplices are very small. We will also triangulate $D^{3}$ as follows: Add a new vertex, $\tilde{p}$, at the center of $D^{3}$ and triangulate $D^{3}$ as the cone over the triangulation of $S^{2}$ with vertex at $\tilde{p}$.

Since $\varrho: S^{2} \longrightarrow M^{n}$ is homotopically non-trivial, we cannot hope to extend it to a disc, thus as an obstruction to the extension we are going to obtain another sphere, which will be in some ways better than the original sphere.

1. Extending a map to 0-skeleton:

Let $\tilde{\varrho}$ take $\tilde{p}$ to $p$, where $p$ is any point on a manifold.

2. Extending the map to 1-skeleton:

We extend the map $\varrho$ to 1 -skeleton by letting $\tilde{\varrho}$ take edges of the form $[\tilde{p}, \tilde{v}]$ to geodesic segments joining $p$ with $\varrho(\tilde{v})=v$. Let us denote those segments by $[p, v]$.

3. Extending the map to 2-skeleton:

We extend the map $\varrho$ to 2 -skeleton by letting $\tilde{\varrho}$ take simplices of the form $\left[\tilde{p}, \tilde{v}_{1}, \tilde{v}_{2}\right]$ to the surfaces generated by special homotopies explained below contracting curves of the form $\left[p, v_{1}\right] \cup\left[v_{1}, v_{2}\right] \cup\left[p, v_{2}\right]$ to the point.

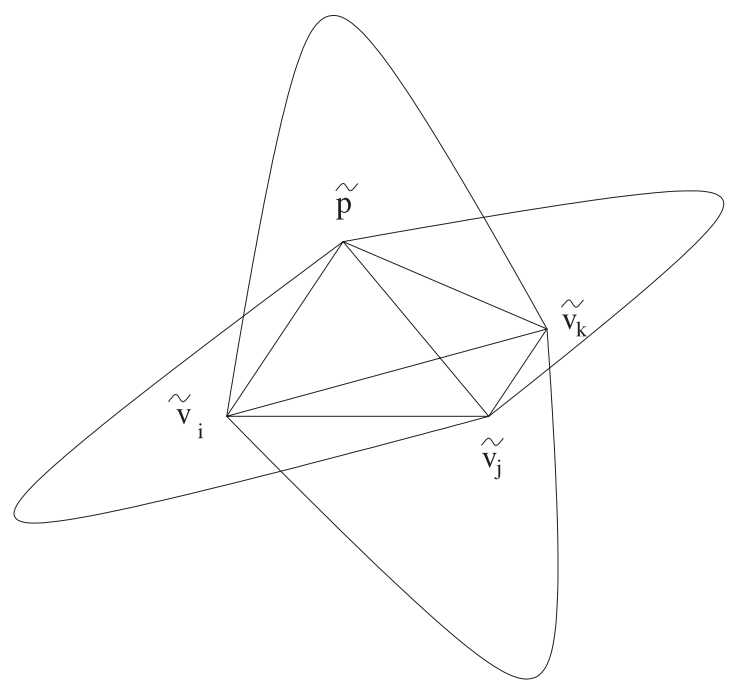

Fig. 4. A sphere of a special shape

Figure 4 illustrates what the image of the boundary of every 3-simplex in the triangulation of $D^{3}$ will look like: It will be made of four surfaces ("tentacles") formed by homotopies contracting boundaries of four 2-dimensional simplices to a point in $M^{n}$. Special properties of the used homotopies will make these tentacles short in the sense that will be made clear later, and so spheres that have this special shape will be called short-tentacled spheres. At least one of those 2-spheres should be non-trivial, otherwise we could extend $\varrho$ to 3 -skeleton, which would be a contradiction. 
In general, for an arbitrary $q$ we will perform a similar procedure, as a result of which we will obtain a short-tentacled sphere of dimension $q$, and in parallel to the case $q=2$, the size of that sphere will depend on the properties of the homotopies that connect spheres of dimension $q-1$ and a point.

Definition 1.1 (Width of Homotopy, (see [SW])). Let $F_{\tau}(t)$ be a homotopy that connects two closed curves parametrized by $t \in[0,1]$ on a Riemannian manifold $M$. We say that $W_{F}$ is the width of the homotopy $F_{\tau}(t)$ if $W_{F}=\max _{t \in[0,1]}$ length of the curve $F_{\tau}(t)$. That is $W_{F}$ is the maximal length of the trajectory described by a point of one of the original closed curves during the homotopy. More generally if $X, Y$ are metric spaces and $F: X \times[0,1] \longrightarrow Y$ is a homotopy then $W_{F}$ is defined as $\sup _{x \in X}$ length of $F(x, *)$.

Definition 1.2 (Short-tentacled sphere). We will say that $\phi: S^{k}=\partial \sigma^{k+1} \longrightarrow M^{n}$ is a short-tentacled sphere of dimension $k$ if one dimensional simplices of $\partial \sigma^{k+1}$ are mapped to minimal geodesics between the images of vertices, and every simplex of dimension at least two is mapped by a special homotopy described below in the paper contracting its boundary.

Observe that the restriction of $\phi$ to the boundary of every simplex of any dimension $>1$ is also a short-tentacled sphere. The only property of special homotopies in this definition that is important to us is that their widths are bounded in terms of the geometric parameters of the manifold, such as the upper bound on volume in Case A and both lower bound on volume and the upper bound on diameter in Case B. We will refer to such special homotopies as "thin".

(ii) How to decompose a short-tentacled sphere into spheres of one dimension lower, so that each point on that sphere describes a short trajectory? In other words, given the short-tentacled sphere $\phi: S^{q} \longrightarrow M^{n}$ constructed in (i) we would like to obtain a map $F:[0,1] \longrightarrow \operatorname{Map}\left(S^{q-1}, M^{n}\right)$ with the properties discussed above.

This step can be illustrated using the following series of pictures, (see Fig. 16 and Fig. 17).

For instance, consider a short-tentacled sphere $\phi: S^{3}=\partial \sigma^{4} \longrightarrow M^{n}$ of dimension 3, where $\sigma^{4}=\left[w_{1}, w_{2}, w_{3}, w_{4}, w_{5}\right]$. Then $\partial \sigma^{4}=\sum_{j=1}^{5}(-1)^{j-1}\left[w_{1}, \ldots, \hat{w}_{j}\right.$, $\left.\ldots, w_{5}\right]$. Each face $\left[w_{1}, \ldots, \hat{w}_{j}, \ldots, w_{5}\right]$ is mapped to the surface generated by contracting its boundary to a point. Let us begin with one of the faces $\sigma_{1}^{3}=\left[w_{1}, \ldots, w_{4}\right]$. This is a 3-simplex and we can "decompose" it into 2-spheres in the obvious way. We know that it is generated by contracting its boundary to a point. Let us call this homotopy $H_{\tau}^{2} . H_{0}^{2}\left(S^{2}\right)=\partial \sigma_{1}^{3} ; H_{1}^{2}\left(S^{2}\right)=v_{0}$. Define $\tilde{H}_{\tau}^{2}=H_{1-\tau}^{2}$. For each fixed $\tau \tilde{H}_{\tau}\left(S^{2}\right)$ is a 2 -sphere. Note that $\tilde{H}_{1}^{2}\left(S^{2}\right)=\partial \sigma_{1}^{3}$ and the width of $\tilde{H}_{\tau}^{2}$ is the same as the one of $H_{\tau}^{2}$, (see Fig. 5).

Next, consider other faces of dimension 3, namely $\sigma_{2}^{3}, \sigma_{3}^{3}, \sigma_{4}^{3}, \sigma_{5}^{3}$ and all faces of dimension $2, \sigma_{i}^{2}=\sigma_{i}^{3} \cap \sigma_{1}^{3}$. For each $\partial \sigma_{i}^{3}$ there exists a homotopy of "small" width that connects it with a point $p$ denoted as $h_{\tau}^{2, i}$. 

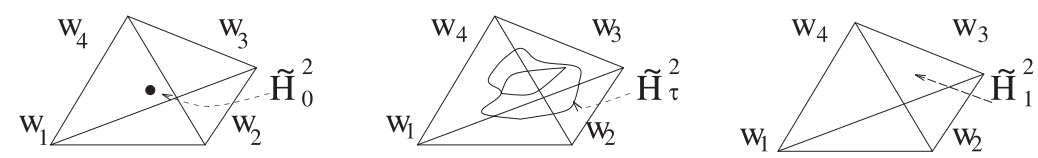

Fig. 5. Decomposing $\left[w_{1}, w_{2}, w_{3}, w_{4}\right]$ into 2-spheres

Then there is a homotopy that joins $\partial \sigma_{1}^{3}$ and the following sphere $S_{\tau^{*}}^{2}$ : instead of $\sigma_{i}^{2}$ consider the following disc $D s_{i}^{2}$ that has the same boundary as $\sigma_{i}^{2}$ and that is obtained by taking the annulus generated by $\left.h_{\tau}^{2, i}\right|_{\tau \in\left[0, \tau^{*}\right]}\left(\partial \sigma_{i}^{2}\right)$ together with the $\operatorname{disc} h_{\tau^{*}}^{2, i}\left(\sigma_{i}^{2}\right) . D s_{i}^{2}$ 's can be naturally glued by the boundary. $S_{\tau^{*}}^{2}$ is the result of this glueing, (see Fig. 6).
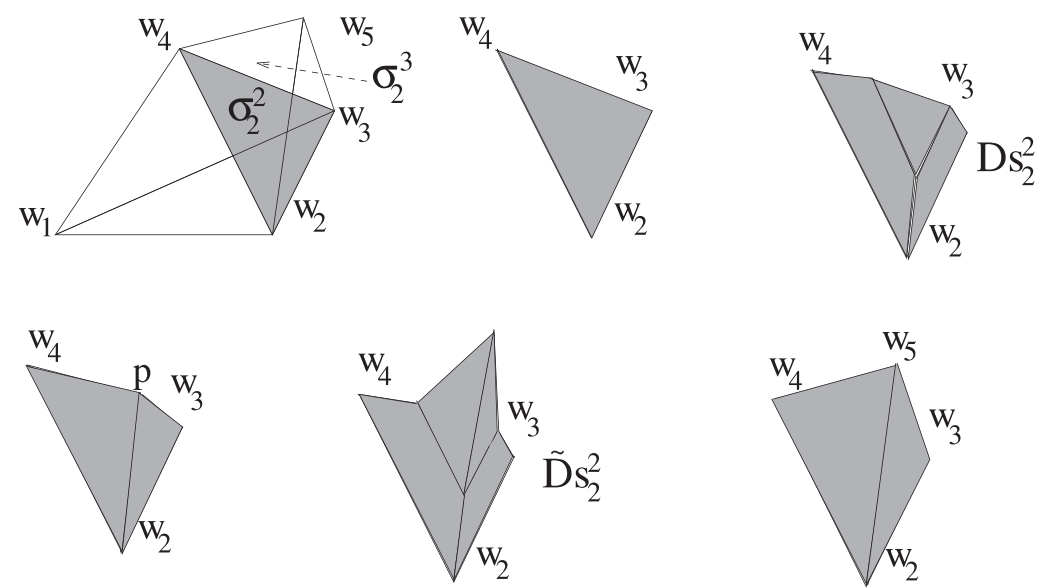

Fig. 6. $D s_{2}^{2}, \tilde{D} s_{2}^{2}$

Now, consider a disc $\tilde{D} s_{i}^{2}$ that has the same boundary as before and is obtained by glueing the annulus $\left.h_{\tau}^{2, i}\right|_{\tau \in[0,1-\tilde{\tau}]}\left(\partial \sigma_{i}^{2}\right)$ and a disc that is obtained as $h_{\tilde{\tau}}^{2, i}\left(\partial \sigma_{i}^{3}-\right.$ $\left.\sigma_{i}^{2}\right), \tilde{\tau} \in[0,1]$, (see Fig. 6).

Now note that combining the above three homotopies parametrized correspondingly by $\tau, \tau^{*}, \tilde{\tau}$ we obain a homotopy between a point $p$ and a 2 -sphere $\left(\partial \sigma_{2}^{3}-\sigma_{2}^{2}\right) \cup\left(\partial \sigma_{3}^{3}-\sigma_{3}^{2}\right) \cup\left(\partial \sigma_{4}^{3}-\sigma_{4}^{2}\right) \cup\left(\partial \sigma_{5}^{3}-\sigma_{5}^{2}\right)$. This 2 -sphere can be contracted to the vertex $w_{5}$ by "cancelling" simplices $\sigma_{i j}^{2}=\partial \sigma_{i}^{3} \cap \partial \sigma_{j}^{3}, i, j \in 2,3,4,5$, each of which occurs twice with the opposite orientation.

(iii) How to construct thin homotopies? This question is the main point of the present paper. As it was stated before, we will consider two different cases: The first case is that of a manifold of curvature and of volume bounded from above, and the second case is that of a manifold $M^{n} \in \mathcal{M}_{-1, v}^{d}$. In the first case, Klingenberg's lemma asserts that the injectivity radius of a compact Rieman- 
nian manifold with sectional curvature bounded above is greater than or equal to $\min \left\{l\left(M^{n}\right) / 2, \pi / \sqrt{K}\right\}$, where $l\left(M^{n}\right)$ is the length of a shortest closed geodesic on a manifold $M^{n}$. It allows us to assume right away that the injectivity radius is bounded from below by $\pi$, (see [GoHL]), (Otherwise, $l\left(M^{n}\right) \leq 2 \pi$ and we are done.)

In the second case, the work of Grove and Petersen gives us the lower bound for the radius of contractibility, (see [GrP]).

The knowledge of either radius of injectivity or radius of contractibility enables us to construct a triangulation tower of a manifold, a notion that generalizes that of a regular triangulation of a manifold, but is somewhat weaker. The reason we use this notion is that geometrical data available to us in the second case is not sufficient to estimate the number of simplices in a triangulation of $M^{n}$. However, the nerve of a covering of $M^{n}$ by metric balls of a controllable radius with centers at a minimal controllably dense net captures a significant part of topology of $M^{n}$ ([GP]). It turns out that for our goals it is convenient to use a tower of nerves of coverings with the same centers but of larger and larger radii which however are less than the radius of contractibility. The numbers of simplices in these nerves can be estimated and their collection (called triangulation tower) turns out to be almost as adequate for us as the usual triangulation. To clarify our ideas first we will assume that $M^{n}$ is endowed with a usual triangulation.

1.2. Construction of homotopies of controlled width: simplicial case. Assume that $M^{n}$ is endowed with a triangulation into small simplices, and we know the upper bound for the number of simplices and (small) upper and (positive) lower bounds for the lengths of 1-dimensional simplices of the triangulation.

As it was stated before, from the technical point of view the main goal is to construct an optimal homotopy, i.e. a homotopy of controlled width, for a sphere that is known to be contractible.

The procedure that we develop in order to construct a homotopy that connects a sphere with a point can be viewed as an effectivisation of Hurewicz Theorem.

1.2.1. The proof of Hurewicz Theorem uses two basic facts:

(a) Any $q$-connected cellular complex is homotopically equivalent to the space with one 0 -dimensional cell and no cells of dimension $i \leq q$.

(b) $i$ th homotopy group is abelian for $i>1$.

We will need quantitative analogues of these statements for our proof. We will use an induction procedure in order to contract simplicial spheres.

1.2.2. The first step in this induction procedure is to learn how to contract 1 dimensional spheres, so that the width of the homotopy $h_{\tau}^{1}$ is bounded in terms of the parameters of a manifold, and so that the surface generated by this homotopy is "built" of "controlable" number of simplices. That procedure was essentially developed in [R]. Here we can use the assumption that every closed curve in $M^{n}$ up to a sufficiently large length can be contracted to a point by a homotopy that does not increase the length of the curve (otherwise, we will immediately get a short closed geodesic), but we do not have a control over the width of this homotopy. 
1.2.3. Now assume that we know how to construct a thin homotopy that connects a simplicial sphere of dimension $\leq k$ to a point. (Here we assume that $1 \leq k<q$ $=\min \left\{i \mid \pi_{i}(M) \neq 0\right\}$.) Assume also that this thin homotopy is simplicial and the surface it generates is composed of a controllable number of simplices. Let us consider a contractible simplicial sphere $s^{k+1}$ of dimension $(k+1)$ that lies in the $(k+1)$-skeleton of $M^{n}$, and assume that we know an upper bound for the number of simplices in this simplicial sphere. We can also view this sphere as a $(k+1)$-chain $C_{k+1}=\sum_{i=1}^{n_{k+1}} a_{k+1}^{i} \sigma_{i}^{k+1}$.

We know that the above chain bounds a chain $C_{k+2}$.

The two key ideas are the following:

1.2.3.1. Since we know the number of simplices that compose the original chain it is possible to estimate the number of simplices of which an optimal $C_{k+2}$ is composed. Indeed, consider the boundary operator as a linear operator from the linear space of $(k+2)$-dimensional chains with real coefficients to the space of $(k+1)$-dimensional chains. In order to represent the considered $(k+1)$-dimensional chain as the boundary of a $(k+2)$-dimensional chain with integer coefficients, we need to find an integer solution of the corresponding linear system. We know that an integer solution of this system exists. All entries of the matrix of the system and the right hand side are integer, and we know an upper bound for their absolute values. Therefore there exists a "small" integer solution, i.e. an integer solution where absolute values of all entries can be estimated. The last assertion is an exercise in linear algebra, see Sect. 4 below for the details.

1.2.3.2. One can use this optimal $C_{k+2}$ in order to construct the required homotopy. Informally and somewhat imprecisely the idea can be explained as follows: Contract the $k$-skeleton of $M^{n}$ to a point. Then the $(k+1)$-skeleton will become a wedge of $k$ spheres. The $(k+2)$ chain becomes a collection of discs bounded by these $k$-spheres. Now we can start from the boundary $(k+1)$-sphere $s^{k+1}$ and remove one by one the $(k+2)$-dimensional cells forming the $(k+2)$-chain by successive homotopies. At the end we will find a homotopy of the initial sphere that bounds $C_{n+2}$ into a "sum" of "basic" $(k+1)$-dimensional spheres, each of which corresponds to a $(k+1)$-dimensional simplex of $M^{n}$. However, each of these spheres can be, in principle, encountered many times with different positive and negative coefficients, and the sum is equal to zero when regarded as a $(k+1)$-dimensional chain. So, for each "basic" $(k+1)$-dimensional sphere $S$ we can permute spheres in this sum combining all occurrences of $S$ with different coefficients together, and then cancel all terms corresponding to $S$ by a homotopy.

More formal explanation of (1.2.3.2): Having now constructed a chain $C_{k+2}$ that has as its boundary $C_{k+1}$ we will proceed as follows:

1.2.3.2.1. We want to use the chain $C_{k+2}$, in order to construct a homotopy $h_{\tau}^{k+1}$ that connects the $(k+1)$-dimensional sphere $s^{k+1}$ with a point. Of course, we know how to contract each simplex $\sigma_{i}^{k+1}$ in that chain with a thin homotopy. The width of $h_{\tau}^{k+1}$ will depend on the number of simplices in the chain $C_{k+2}$, taken with their multiplicities, the width of contracting a single simplex and on the width of contracting spheres of lower dimension. 
We will begin with the following:

1.2.3.2.2. We have already noted that a $(q-1)$-connected manifold is homotopically equivalent to a $\mathrm{CW}$-complex with one 0 -dimensional cell and no cells of dimension $1 \leq i \leq(q-1)$. Assuming that our manifold has been triangulated, $q$-skeleton of this CW-complex will consist of a wedge of spheres of dimension $q$ with one sphere for each simplex of $M^{n}$. We will denote this complex $C W_{(q-1)}$. Let us also consider complexes $C W_{i}$ for $0 \leq i \leq q-1$, which are obtained from $M^{n}$ by identifying the $i$-skeleton of $M^{n}$ to a point.

We are going to describe maps $\tilde{g}_{i}: C W_{i} \longrightarrow M^{n}$. First of all let us point out that the only 0 -dimensional cell of $C W_{i}, \tilde{v}_{0}$ will always be mapped to $v_{0}$, a preselected vertex of $M^{n}$.

Now let us consider 1-skeleton of $C W_{0}$. It will be a wedge of circles: one circle for each edge of $M^{n}$. Consider a circle that corresponds to the edge $e_{i j}$ that connects vertices $v_{i}$ and $v_{j}$. For each vertex $v_{i}$ fix a shortest path $P_{i}$ that connects this vertex with $v_{0}$ and lies in 1-skeleton of $M^{n}$. The circle will be mapped to $P_{i} \cup e_{i j} \cup-P_{j}$, where $P_{i}, P_{j}$ are paths that lie in 1-skeleton of $M^{n}$ and that connect vertices $v_{i}, v_{j}$ to $v_{0}$. This map can be extended to the whole of $C W_{0}$. For instance, consider the 2-skeleton of $C W_{0}$. Each 2-cell of $C W_{0}$ is the image of a 2-simplex of $M^{n}$. Without loss of generality consider a 2-cell that is the image of $\sigma^{2}=\left[v_{1}, v_{2}, v_{3}\right]$ and view it as a union of the "inside" disc and an "outside" annulus glued along their common boundary. Map the disc onto the simplex. The outer boundary of the outside annulus is mapped using the 3 maps of the three closed 1-cells of $C W_{0}$. The annulus is mapped using a homotopy between the maps of its inner and outer boundaries.

Next, consider the 2-skeleton of $C W_{1}$. This is a wedge of 2-spheres corresponding to each 2-simplex $\sigma^{2}$ of $M^{n}$. (Each 2-sphere in the wedge is the image of a 2-simplex of $M^{n}$.) Then we will let the image of any such 2-sphere be the simplex, (that is its inverse image) together with the disc generated by a previously fixed homotopy that connects the boundary of this simplex with $v_{0}$. It is essential in this case that all the homotopies that contract (simplicial) 1 -spheres with $v_{0}$ have been fixed and if two simplices $\sigma_{1}^{2}, \sigma_{2}^{2}$ share a face then those homotopies (for the boundaries of $\sigma_{1}^{2}, \sigma_{2}^{2}$ ) will agree, when restricted to this face. This scheme is illustrated on Fig. 7. Here $S F_{i}^{2}$ for $i=1,2,3$ denotes a surface generated by a homotopy that contracts the closed curve that consists of the edge $e_{i}$ in the boundary of $\sigma^{2}$ together with two curves that join corresponding verteces with $v_{0}$ to a point.

To extend this map to the 3-skeleton of $C W_{1}$ we consider a 3-cell, which is the image of a 3 -simplex $\sigma^{3}=\left[v_{1}, v_{2}, v_{3}, v_{4}\right]$. As in the previous case, we can view this cell as the union of the inside disc and the outside annulus. We will map the disc onto the simplex $\sigma^{3}$; the outer boundary of the outside annulus is mapped using the 4 maps of cells of $C W_{1}$. The annulus is mapped using a homotopy between the maps of its inner and outer boundary. This homotopy is just the extension of the homotopies $\tilde{h}_{\tau}^{1}$ contracting boundaries of 1 -dimensional cells $\tilde{\sigma}^{1}$ in the boundary of $\tilde{\sigma}^{2}$. Similarly, to extend this map to the $k$-skeleton consider a $k$-cell, the image of $k$-simplex $\sigma^{k}=\left[v_{1}, \ldots, v_{k+1}\right]$. Subdivide this cell into a disc and the annulus, map 

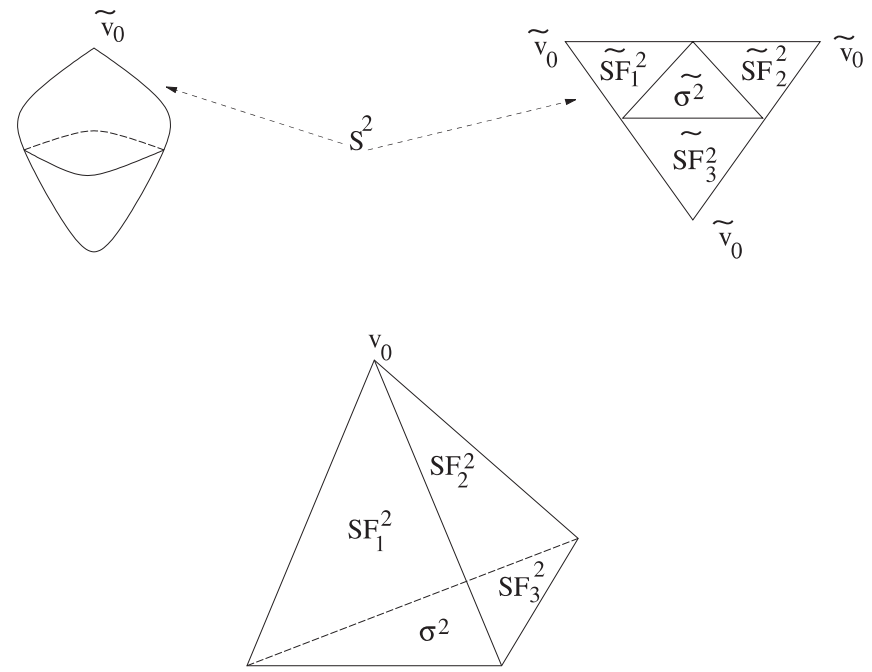

Fig. 7. The map from $S^{2}$ to the manifold

the disc onto the $k$-simplex $\sigma^{k}$. Then the outer boundary of the annulus is mapped using the maps of cells of $C W_{1}$, and the annulus is mapped using a homotopy between the maps of its inner and outer boundary.

Similarly, we can deal with the rest of $\tilde{g}_{i}^{\prime} s$. That is, consider a complex $C W_{i-1}$. Its $i$-skeleton is a wedge of $i$-spheres: a sphere for each $i$-simplex of $M^{n}$. Interpret this sphere as the boundary of an $(i+1)$-dimensional simplex and denote one of the faces $\tilde{\sigma}^{i}$. Then we will map $\tilde{\sigma}^{i}$ to the simplex $\sigma^{i}$ to which this sphere corresponds. Next consider the boundary of $\sigma^{i}$. For each of the faces of the boundary there is already a fixed homotopy of its boundary to a point. (Here we are using the induction assumption.) This homotopy together with the face forms a sphere. Denote the subset generated by a homotopy that connects this sphere with a point by $S F_{j}^{i}$. Now our original sphere $\tilde{S}^{i}$ will be mapped to the sphere formed by $S F_{j}^{i}, j=1,2, \ldots, i+1$ together with $\sigma^{i}$. This map can be extended to skeleta of $C W_{i_{1}}$ of higher dimensions as in the previous cases.

The above considerations enable one to "view" $M^{n}$ as a singular complex with one cell of dimension 0 , and no cells of dimension $1 \leq i \leq(q-1)$. To illustrate this observe that if $q=1$ we can view a 1-simplex $e_{i j}$ as a closed 1-cell $c l_{i j}=P_{i} \cup e_{i j} \cup-P_{j}$.

1.2.3.2.3. Now, in order to contract a sphere $s^{k+1}$, we can first map it to $C W_{k}$, note that there it is homologous to zero, thus bounds a disc, by Hurewicz theorem; use the optimal chain $C_{k+2}$ that it bounds to construct an explicit homotopy, then transfer everything back to the manifold using $\tilde{g}_{k}$ and note that the width will depend on the number of simplices in the chain $C_{k+2}$ (taken with their multiplicities) and the width of previous homotopies. However, in order to make the width of the homotopy of the sphere in $M^{n}$ independent of the number of simplices in $s^{k+1}$ we 


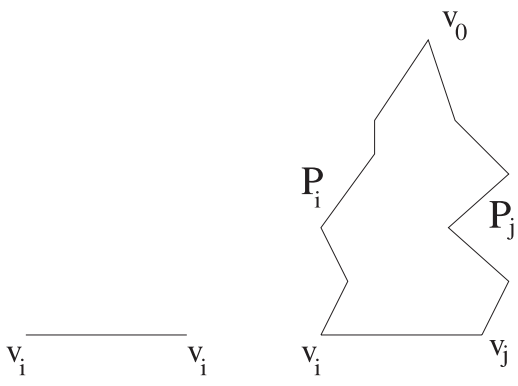

Fig. 8. The "transformation" of a 1-simplex

first represent $s^{k+1}$ as a sum of simplicial spheres with a controllable number of simplices and contract them using $C W_{k}$ and cobounding chains as described above. (The number of these spheres is equal to the number of $(k+1)$-simplices of $s^{k+1}$. Here we use a homotopy of controlled width contracting the $k$-skeleton of $s^{k+1}$. See Sect. 5 for more details.)

1.3. Construction of homotopies of controlled width: the general case. However, the above does not explain what one can do if the radius of convexity is not known and there is no way to construct a triangulation of $M^{n}$ where the number of simplices can be estimated in terms of the parameters of $M^{n}$.

Suppose $M^{n} \in \mathcal{M}_{-1, v}^{d}$. We will use the result of Grove and Petersen that allows us to estimate the radius of contractibility $r$ on such a manifold and (important!) the width of a homotopy contracting a metric ball of radius $x<r$ to a point inside the concentric metric ball of radius $C x$, where $C=C(n, v, d)$ also can be estimated. As an example, we will describe how to construct a thin homotopy for a sphere of dimension 2 that connects it with a constant map.

We will begin by covering $M^{n}$ by balls of radius much smaller than the radius of contractibility. The number of such balls can be estimated. Next, we consider the $(n+1)$-skeleton of the nerve of this cover and call it $\mathcal{K}_{1}$. Increase radii of balls by some factor explained later, keeping their centers fixed and consider the $(n+1)$-skeleton of the nerve $\mathcal{K}_{2}$ of that cover. There is a natural embedding of $\mathcal{K}_{1}$ to $\mathcal{K}_{2}$. Now, we increase the radii of balls by the same factor one more time and consider $\mathcal{K}_{3}$ of the nerve of this cover. Again, there will be a natural embedding of $\mathcal{K}_{2}$ to $\mathcal{K}_{3}$. We choose the radii of the balls of the cover so that all small spheres in $\mathcal{K}_{i}$ are contractible in $\mathcal{K}_{i+1}$. Since $M^{n}$ is $(q-1)$-connected all spheres up to dimension $(q-1)$ in $\mathcal{K}_{i}$ are contractible in $\mathcal{K}_{i+1}$. (Here we use the same trick as throughout the paper: We can map the sphere in $\mathcal{K}_{i}$ to $M^{n}$, contract it in $M^{n}$, then map it back to $\mathcal{K}_{i}$ and obtain there a new sphere that is contractible in $\mathcal{K}_{i}$. This new sphere will be different from the original one, however the distance between them will be small enough for them to be homotopic in $\mathcal{K}_{i+1}$ ). However, we need a control over parameters of this homotopy and therefore prefer to use several nerves. Note also, that we have maps $M^{n} \longrightarrow \mathcal{K}_{i}$ and $\mathcal{K}_{i} \longrightarrow M^{n}$ that enable us to replace the problem of contracting spheres in $M^{n}$ by its simplicial analog. The first of these maps is the composition of the map to the nerve $\mathcal{K}_{1}$, a simplicial 
approximation, and the inclusion $\mathcal{K}_{1} \longrightarrow \mathcal{K}_{i}$. The second map can be constructed by an induction procedure explained below. The key idea of this procedure is that if we already have a map of the boundary of a simplex into a small ball in $M^{n}$, then it can be extended to a "nice" map of the simplex into a somewhat larger but still small ball in $M^{n}$ using the local contractibility properties of $M^{n}$ as in [GrP], [P2].

First, let us explain how to contract a simplicial 2-sphere that is in $\mathcal{K}_{1}$. Denote this sphere by $\tilde{S}^{2}$ and denote the map of $\mathcal{K}_{i}$ to $M^{n}$ by $g_{i}$ and the map going in the other direction by $f_{i}$. The image $S^{2}$ of $\tilde{S}^{2}$ in $M^{n}$ is contractible in $M^{n}$, assuming $M^{n}$ is $(q-1)$-connected and $q \geq 3$. Therefore, the image $\bar{S}^{2}$ of $S^{2}$ is contractible in $\mathcal{K}_{1}$.

$\bar{S}^{2}$ does not coincide with $\tilde{S}^{2}$, but $g_{1}$ and $f_{1}$ can be selected in such a way that they don't lie far from each other. Thus, they are homotopic in $\mathcal{K}_{2}$. So, we see that a sphere $\tilde{S}^{2} \subset \mathcal{K}_{1}$ is contractible in $\mathcal{K}_{2}$.

However, in order to get a good control over the width of the contracting homotopy (indepent of the number of simplices in $\tilde{S}^{2}$ ) we will contract $\tilde{S}^{2}$ in $\mathcal{K}_{4}$ as follows: First, fix paths connecting all vertices of $\tilde{S}^{2}$ with the base point. Every 1-simplex of $\tilde{S}^{2}$ becomes a simplicial 1-sphere passing through the base point after combining it with the paths connecting its endpoints with the base point. These simplicial 1-spheres can be contracted in $\mathcal{K}_{2}$ by a homotopy of controlled width. Now consider $\tilde{S}^{2}$ as a simplicial sphere in $\mathcal{K}_{2}$. It is homotopic to the sum of 2 -spheres obtained as follows: For any 2-simplex $\sigma$ of $\tilde{S}^{2}$ consider a simplicial sphere obtained from $\sigma$ by adding 3 simplicial discs formed by contracting 3 simplicial 1 -spheres obtained from edges of $\sigma$ as above. (The homotopy between $\tilde{S}^{2}$ and the sum of these spheres will insert pairs of oppositely oriented discs obtained in this way.) So, it is sufficient to learn how to contract each of these spheres in a satisfactory way in order to learn to contract $\tilde{S}^{2}$. Note that the number of simplices in any of these spheres does not depend on the number of simplices in $\tilde{S}^{2}$. Let $\tilde{S}_{0}^{2}$ be one of these spheres. It is contractible in $\mathcal{K}_{3}$. (One can use the argument used to show that $\tilde{S}^{2}$ is contractible in $\mathcal{K}_{2}$ ). Therefore it bounds a chain in $\mathcal{K}_{3}$ and we can estimate the number of simplices in the optimal chain $C$ that it bounds.

Consider the $\mathrm{CW}$-complex, obtained from $\mathcal{K}_{3}$ by identifying its 1-skeleton with a point. Let us denote it by $\operatorname{Comp}_{3,1}$. This CW-complex will have one vertex, no 1-dimensional cells and its 2-dimensional skeleton will be a wedge of 2-spheres with one sphere corresponding to each 2 -simplex of $\mathcal{K}_{3}$. There is a quotient map from $\mathcal{K}_{3}$ to $\operatorname{Comp}_{3,1}$ and one can construct a map from Comp $_{3,1}$ to $\mathcal{K}_{4}$ that will be denoted by $\tilde{\tilde{g}}_{3,1}$ and will be described later on.

The next step is to consider the image ${\tilde{S_{0}}}^{2}$ of ${\tilde{S_{0}}}^{2}$ in $\operatorname{Comp}_{3,1}$. ${\tilde{\tilde{S}_{0}}}^{2}$ bounds a chain, so it is contractible, and, due to the nature of Comp $_{3,1}$ one can construct the contracting homotopy that uses the underlying space of this chain. Now, consider the image of $\tilde{\tilde{S}}_{0}{ }^{2}$ in $\mathcal{K}_{4}$. The map from $\operatorname{Comp}_{3,1}$ to $\mathcal{K}_{4}$ is constructed so that each sphere that is the image of a 2 -simplex in $\mathcal{K}_{3}$ is mapped back to this simplex together with the disc that is generated by the homotopy that connects the boundary of this simplex (regarded as the wedge of three 1-spheres as explained above) with the base point. The image of $\tilde{\tilde{S}}_{0}{ }^{2}$ in $\mathcal{K}_{4}$ will be obviously null homotopic by a homotopy with the desired properties. This image is not ${\tilde{S_{0}}}^{2}$, but we can construct 
a homotopy between this image and $\tilde{S}_{0}^{2}$. Roughly speaking, the image of $\tilde{\tilde{S}}_{0}^{2}$ is $\tilde{S}_{0}^{2}$ plus pairs of oppositely oriented discs contracting simplicial 1-spheres obtained from 1-simplices of $\tilde{S}_{0}^{2}$. The homotopy between $\tilde{\tilde{S}}_{0}{ }^{2}$ and $\tilde{S}_{0}^{2}$ will cancel these pairs of discs.

1.4. Plan of the remaining part of the paper. In Sect. 2 we introduce the triangulation towers mentioned in 1.3. Section 3 starts from the discussion of complexes Comp $_{i, k}$ obtained from $\mathcal{K}_{i}$ by collapsing its $k$-skeleton to a point and their maps to/from $M^{n}$ (see the discussion in 1.2.3.2.2 and 1.3 above). Then we state Proposition 3.2 asserting that if all closed curves in a $(q-1)$-dimensional Riemannian manifold $M^{n}$ of length less than a certain explicit quantity are contractible without length increase, then any contractible simplicial sphere of dimension $k \leq q$ can be contracted by a homotopy of controlled width. Then we take care of the case, $k=1$, establishing the basis for an inductive proof of Proposition 3.2. Section 4 contains a detailed description of the result mentioned in 1.2.3.1: If a $m$-dimensional chain in $M^{n}$ bounds a $(m+1)$-dimensional chain with integer coefficients, then it bounds an $(m+1)$-dimensional chain with integer coefficients such that the sum of absolute values of these coefficients does not exceed a certain explicit quantity depending on $n$, the numbers of $m$-dimensional and $(m+1)$-dimensional simplices in $M^{n}$ and the sum of the absolute values of the coefficients of the given $m$-dimensional chain.

Section 5 starts from the proof of Proposition 3.2 along the lines of the discussion in 1.2.3.2 (with the modifications outlined in 1.3 that take care of the fact that $M^{n}$ is not triangulated but only endowed with a triangulation tower). Then we observe that the width of the contracting simplicial homotopy can be made independent of the number of simplices in the sphere that is being contracted as it was explained in 1.2.3.2.3 (Important Remark 5.2). This observation enables us to complete the construction of maps $\mathrm{Comp}_{i, k} \longrightarrow M^{n}$. (Here we would like to observe that the proof of Proposition 3.2 in Sects. 3-5 has a recursive nature: In order to contract a $k$-dimensional sphere by a homotopy of controlled width we need maps $\operatorname{Comp}_{i, l} \longrightarrow M^{n}$ for all $l<k$, which can be defined only after we will show how to contract all spheres of dimension $\leq l<k$ by such homotopies). Then we extend Proposition 3.2 for $k$-dimensional spheres that are not necessarily simplicial (Theorem 5.2). At the beginning of Sect. 6 we combine Theorem 5.2 and the trick explained in Sect. 1.1(i) above to construct the required short-tentacled spheres. Then we use the idea explained in 1.1(ii) to prove Theorems 0.1 and 0.2 . At the beginning of Sect. 7 we observe that our proofs of Theorem 5.2 and Theorems $0.1,0.2$ immediately imply Theorems $0.3,0.4$. Then we prove Theorem 0.5 . The proof of Theorem 0.5 does not use any material from Sects. 1-6 and can be read independently of the rest of the paper.

\section{Triangulation tower and the nerve of the covering}

In this section we define the notion of a triangulation tower of a Riemannian manifold $M^{n}$, outline a construction of such towers in the situation when the 
contractibility radius and a contractibility function of $M^{n}$ are known and estimate the number of simplices in the constructed triangulation tower.

We will consider two examples of such manifolds, namely, manifolds, with injectivity radius bounded from below, and manifolds with curvature and volume bounded from below, and diameter bounded from above.

Definition 2.1 (Triangulation tower). A triangulation tower of a $(q-1)$-connected manifold $M^{n}$ is the data of a collection of simplicial complexes $\mathcal{K}_{1} \subset$ $\mathcal{K}_{2} \subset \ldots \subset \mathcal{K}_{2 q}$ and maps $f_{i}: M^{n} \longrightarrow \mathcal{K}_{i}, g_{i}: \mathcal{K}_{i} \longrightarrow M^{n}$ that commute with inclusions of $\mathcal{K}_{i}$ 's and such that the composition of $g_{i}$ and $f_{i}$ is homotopic to the identity map $M^{n} \longrightarrow M^{n}$. Moreover, let $k_{i}: \mathcal{K}_{i} \longrightarrow \mathcal{K}_{i+1}$ be the inclusion homomorphism. We require that $k_{i * j}=f_{i+1 * j} \circ g_{i * j}$ for all $j \leq n$, where $k_{i * j}: \pi_{j}\left(\mathcal{K}_{i}\right) \longrightarrow \pi_{j}\left(\mathcal{K}_{i+1}\right) ; f_{i+1 * j}: \pi_{j}\left(M^{n}\right) \longrightarrow \pi_{j}\left(\mathcal{K}_{i+1}\right)$ and $g_{i * j}: \pi_{j}\left(\mathcal{K}_{i}\right) \longrightarrow \pi_{j}\left(M^{n}\right)$ are induced homomorphisms.

Definition 2.2 (The contractibility function). The contractibility function $\varrho$ : $[0, R] \longrightarrow[0, \infty)$ takes $\varepsilon$ to $\varrho(\varepsilon)$ if a ball of radius $\varepsilon$ is contractible inside the ball of radius $\varrho(\varepsilon)$. Here, $R$ is called the radius of contractibility.

For example, if the $\operatorname{injrad}\left(M^{n}\right) \geq \pi$ then $\varrho:[0, \pi] \longrightarrow[0, \infty)$ and $\varrho(\varepsilon)=\varepsilon$. If $M^{n} \in \mathcal{M}_{-1, v}^{d}$ then $\varrho(\varepsilon)=C(n, v, d) \cdot \varepsilon$, where one can take $C=C(n, v, d)=$ $(\exp (d) / \min \{1, v\})^{c(n)}, R=R(n, v, d)=\left(\frac{\min \{1, v\}}{\exp (d)}\right)^{c(n)}$ for some constant $c(n)$. For brevity we will be using the letter $C$ for $C(n, v, d)$. (see $[\mathrm{P}]$, [GrP]).

We will now describe a specific triangulation tower of $M^{n}$ required for our purposes in two cases:

Case A. the case of $M^{n}$, when $\operatorname{injrad}\left(M^{n}\right) \geq \pi$;

Case B. the case of $M^{n} \in \mathcal{M}_{-1, v}^{d}$.

First, we will construct a sequence of four-tuples $\left\{\mathcal{U}_{i}, \mathcal{K}_{i}, f_{i}, g_{i}\right\}_{i=1}^{2^{q}}$, where $\mathcal{U}_{i}$ will be covers of $M^{n}$ by balls of radii $\delta_{i}$ increasing with $i, \mathcal{K}_{i}$ will be the $(n+1)$ skeleton of the nerve of this cover, $f_{1}$ will be a map from the manifold to $\mathcal{K}_{1}$ via a partition of unity subordinate to $\mathcal{U}_{1}$ plus subsequent pushing to the $(n+1)$ skeleton (a specific choice of how to push the image of the map to the nerve to the $(n+1)$-skeleton will not be important for us providing that the image of every point remains in the closure of the original cell in the nerve), and $f_{i}$ will be the composition of $f_{1}$ and the inclusion of $\mathcal{K}_{1}$ into $\mathcal{K}_{i}$. Finally, $g_{i}$ will be constructed later on.

In our construction $\mathcal{U}_{1}$ will be a covering of the manifold by sufficiently small balls of some radius $5 \delta$, chosen appropriately and $\mathcal{U}_{i+1}$ will be obtained from $\mathcal{U}_{i}$ by keeping centers of balls the same as before and increasing their radii by a factor of $\xi_{1}(n)$, in Case A and by a factor of $\xi_{2}(n) \cdot C(n, v, d)^{n+1}$, in Case B, where $\delta$ should be chosen so that the image of any $j$-simplex, where $j \leq n$ from $\mathcal{K}_{i}$ lies within a ball of radius less than $\pi$ in Case $\mathrm{A}$ and the lower bound $R$ for radius of contractibility in Case B. $\xi_{1}(n), \xi_{2}(n)$ are constants that depend on the dimension of a manifold only. 
Since we are not going to evaluate the dependence of upper bounds on $n$ in this paper, we will often denote different constants that depend only on $n$ by the same notation. So $c(n)$ may have different meanings in different parts of the paper (and the same is true for $c_{1}(n), c_{2}(n)$, etc.).

Let us begin by constructing the first covering of $M^{n}: \mathcal{U}_{1}$. First, let's construct a $\delta$-net on our manifold and estimate the number of elements in it, where in

Case $\mathbf{A}$ we let $\delta=\frac{\pi}{c(n)}$; and in

Case $\mathbf{B} \delta=\frac{R}{c_{1}(n) \cdot\left(C^{c_{2}(n)}+1\right)}$.

In order to estimate $n_{0}$, the number of elements in the $\delta$-net, we need to recall certain facts.

Case A. In the first case we will use Croke's inequality: $\operatorname{vol}(B(r, p)) \geq$ const $_{\cdot n} r^{n}$, where $B(r, p)$ is any metric ball of radius $r$ centered at $p \in M^{n}$ and $r<\operatorname{inj}\left(M^{n}\right) / 2$. We can take const $_{\cdot n}=\frac{2^{n-1}}{(n !)^{2}}$, see [Ch].

Using this inequality we can estimate $n_{0}$, the number of elements in the minimal $\delta$-net, by estimating the maximal number of pairwise disjoint metric balls of radius $\frac{\delta}{2}$.

The number of such balls will be less than or equal to $\frac{c(n) \operatorname{vol}\left(M^{n}\right)}{\delta^{n}}$.

Let us call this estimate $N_{0}$.

Case B. In the second case the estimate will be obtained using to the volume comparison theorem, i.e. we have

$$
\frac{\operatorname{volM}^{n}}{\max _{i} \operatorname{vol}_{\delta / 2}\left(v_{i}\right)} \leq \frac{\int_{0}^{d}(\sinh s)^{n-1} d s}{\int_{0}^{\delta / 2}(\sinh s)^{n-1} d s}
$$

from where we obtain $N_{0}=\frac{c(n) e^{n d}}{\delta^{n}}$.

Next, let us cover $M^{n}$ by $\left\{B_{5 \delta}\left(v_{i}\right)\right\}_{i=1}^{n_{0}}$.

We will now proceed to construct $\phi=g_{1}$ by means of the following lemma, which is a particular case of the Main Lemma from [P].

Lemma 2.3. Let $L=\mathcal{K}_{1}$ be the nerve of the covering of $M^{n}$, constructed as above. Consider the two cases:

Case A: the radius of injectivity of $M^{n}$, inj $\left(M^{n}\right)$ is bounded from below by $\pi$.

Case B: $M^{n} \in \mathcal{M}_{-1, v}^{d}$.

Let $L^{0}$ denote the 0 -skeleton of the nerve of the covering. Then the natural map $\phi_{0}: L^{0} \rightarrow M^{n}$ can be extended to a continuous map $\phi: L \longrightarrow M^{n}$ in such a way that if $\tilde{\sigma}^{k} \subset L$ is a k-simplex then

Case A: $\phi\left(\tilde{\sigma}^{k}\right) \subset B(\phi(v), k \delta)$ for any vertex $v$ of $\tilde{\sigma}^{k}$.

Case B: $\left.\phi\left(\tilde{\sigma}^{k}\right) \subset B(\phi(v)), 3^{k} \frac{\delta\left(C^{k+1}-1\right)}{C-1}\right),($ see $[P])$. 
Proof. Once again, the construction is by induction on skeleton of $L$.

\section{1-skeleton.}

Let $\phi\left(\tilde{e}_{i j}\right)=e_{i j}$, where $e_{i j}$ is a minimizing geodesic that connects $v_{i}$ and $v_{j}$.

\section{2-skeleton.}

Consider $\tilde{\sigma}_{i j k}^{2}$ with the boundary $\partial \tilde{\sigma}_{i j k}^{2}=\tilde{e}_{i j} \cup \tilde{e}_{j k} \cup \tilde{e}_{k i}$. By the first step, we know that $\phi\left(\partial \tilde{\sigma}_{i j k}^{2}\right)=e_{i j} \cup e_{j k} \cup e_{k i}$, so $\phi\left(\partial \tilde{\sigma}_{i j k}^{2}\right) \subset B(v, 2 \delta)$, so, in Case A, it can be contracted to $v$ along the minimal geodesics that join $v$ and $\phi\left(\partial \tilde{\sigma}_{i j k}^{2}\right)$, and in Case $\mathrm{B} B(v, 2 \delta)$ is also contractible to $v$ inside the ball of a larger radius, so $\phi\left(\partial \tilde{\sigma}_{i j k}^{2}\right)$ can be contracted to $v$ as well. Then we can map $\tilde{\sigma}_{i j k}^{2}$ using this homotopy.

The rest follows by induction:

Let $\tilde{\sigma}^{k}\left(\tilde{v}_{0}, \ldots, \tilde{v}_{k}\right)$ be a $k$-simplex and assume $\phi\left(\partial \tilde{\sigma}^{k}\right) \subset B(v, k \delta)$ in Case A, and $\phi\left(\partial \tilde{\sigma}^{k}\right) \subset B\left(v, 2\left(3^{k-1}+3^{k-2}\right) \frac{\delta\left(C^{k}-1\right)}{C-1}\right)$ in Case B. Then $\phi\left(\partial \tilde{\sigma}^{k}\right)$ can be contracted to a vertex $v$ by either continuously connecting $v$ with $\phi\left(\partial \tilde{\sigma}^{k}\right)$ with minimal geodesics in Case A, or by connecting it with $v$ with the trajectories of the points of $\phi\left(\partial \tilde{\sigma}^{k}\right)$ under the contraction of the ball. We then extend $\phi$ by letting the image of $\tilde{\sigma}^{k}$ be the set generated by the homotopy above.

The construction of $\left(\mathcal{K}_{i}, g_{i}\right)$ is analogous to the above.

In order to pass from $\mathcal{K}_{i}$ to $\mathcal{K}_{i+1}$ we increase the radii of the balls of the cover of $M^{n}$ used to construct $\mathcal{K}_{i}$ by a factor which is chosen so that all "small" spheres in $\mathcal{K}_{i}$ will be contractible in $\mathcal{K}_{i+1}$, and, as the result, the composition of maps of $\mathcal{K}_{i}$ to $M^{n}$ and back will be $n$-homotopic to identity in $\mathcal{K}_{i+1}$. (The meaning of the expression " $n$-homotopic to identity" is that the restriction of this map to the $n$-skeleton is homotopic to identity. The necessity to use this notion here is due to the fact that $K_{i}$ 's were defined not as nerves of the coverings but as $(n+1)$-dimensional skeleta of these nerves.) More specifically it is not difficult to see that we can take the radii of the balls used to construct $\mathcal{K}_{i+1}$ to be $c(n) C(n, v, d)^{n+1}$ times as large as the radii of the balls used to construct $\mathcal{K}_{i}$, where $C(n, v, d)$ is the contractibility constant in Case B. In Case A, we can take radii of the balls used to construct $\mathcal{K}_{i+1}$ to be equal to the radius of the balls that are used to construct $\mathcal{K}_{i}$ multiplied by $n+1$.

Remark. To give a partial explanation why we need $2^{q}$ nerves in the definition of a triangulation tower note that although any simplicial sphere of dimension $i \leq(q-1)$ from $\mathcal{K}_{1}$ can be contracted in $\mathcal{K}_{2}$ we need a control over the number of simplices in a contracting simplicial homotopy. In order to obtain this control we devised an inductive procedure described below, when in order to contract a simplicial sphere in $\mathcal{K}_{1}$ of dimension $i$ effectively, we first learn how to effectively contract simplicial spheres of all dimensions $<i$ using nerves with larger and larger indices. Using this procedure, one can effectively contract a sphere of dimension $i \leq(q-1)$ only in $\mathcal{K}_{2^{q}}$ (see below).

\section{Thin homotopies of $k$-dimensional simplicial spheres}

In the next three sections we will develop the procedure for a construction of a homotopy of a simplicial $k$-dimensional sphere $S^{k}$ to a point, such that the width 
of this homotopy will be bounded in terms of an upper bound on the volume of a manifold $M^{n}$ in Case A and in terms of a lower bound on the volume and an upper bound on the diameter in Case B.

This procedure will be inductive on the dimension of the sphere.

First, we will give a proof of a version of this statement using the notion of triangulation tower and useful for our purposes.

Definition 3.1 (Singular cell subdivision sequence). Given a triangulation tower $\mathcal{K}_{1} \subset \mathcal{K}_{2} \subset \ldots \subset \mathcal{K}_{2 q}$, for each $\mathcal{K}_{i}$ we can construct a singular cell subdivison sequence, which will be a sequence of triplets $\left\{\operatorname{Comp}_{i, k}, \tilde{f}_{i, k}, \tilde{g}_{i, k}\right\}_{k=0}^{i-1}$, where Comp $\operatorname{Com}_{i, k}$ will be $\mathcal{K}_{i}$ with its $k$-skeleton identified to a point, $\tilde{f}_{i, k}=\pi_{i, k} \circ f_{i}: M^{n} \rightarrow$ Comp $_{i, k}$, where $\pi_{i, k}: \mathcal{K}_{i} \longrightarrow$ Comp $_{i, k}$ is a quotient map and the construction of $\tilde{g}_{i, k}:$ Comp $_{i, k} \longrightarrow M^{n}$ will be described below.

The construction of $\tilde{g}_{i, k}$. We will now define $\tilde{g}_{i, k}:$ Comp $_{i, k} \longrightarrow M^{n}$ by first defining a map $\tilde{\tilde{g}}_{i, k}: \operatorname{Comp}_{i, k} \longrightarrow \mathcal{K}_{i-1+2^{k}}$ and then composing it with $g_{i-1+2^{k}}$.

In order to define $\tilde{\tilde{g}}_{i, k}$ first we need to introduce simplicial homotopies $\tilde{h}_{i, l, \sigma^{l}}$ that contract certain simplicial spheres of dimension $l \leq k$ from $\mathcal{K}_{i+2^{l-1}-1}$ in $\mathcal{K}_{i+2^{l}-1}$. These spheres are in a bijective correspondence with $l$-dimensional simplices $\sigma^{l}$ of $\mathcal{K}_{i}$.

Fix the shortest simplicial paths connecting each vertex of $\mathcal{K}_{1}$ with the base point in $\mathcal{K}_{1}$. Let $l=1$. Then we assign to a 1 -dimensional simplex $\sigma_{1}$ the 1-dimensional simplicial sphere obtained from $\sigma_{1}$ by attaching the simplicial paths connecting its endpoints with the base point. The resulting simplicial 1-sphere can be contracted in $\mathcal{K}_{i+1}$ by a simplicial homotopy described below in Lemma 3.3. The homotopies $\tilde{h}_{i, l, \sigma^{l}}$ for all $l \leq k$ will be constructed later in Lemma 5.1. The construction of $\tilde{h}_{i, l, \sigma^{l}}$ uses the construction of maps $\tilde{\tilde{g}}_{j, m}: \operatorname{Comp}_{j, m} \longrightarrow \mathcal{K}_{j+2^{m}-1}$ for all $j$ and for all $m<l$. So, we obtain an inductive "bootstrap" procedure defining $\tilde{h}_{i, k, \sigma^{k}}$ and $\tilde{\tilde{g}}_{i, k}$ that starts from Lemma 3.3 below. Now we are going to describe how to construct $\tilde{\tilde{g}}_{i, k}$ if $\tilde{h}_{i, l, \sigma^{l}}, l \leq k$, are already constructed.

First, we are going to explain which simplicial sphere is going to be contracted by $\tilde{h}_{i, l, \sigma^{l}}$. Connect all vertices of $\sigma^{l}$ with the base point by the fixed simplicial paths. Then every 1-dimensional simplex $\sigma^{1} \subset \sigma^{l}$ becomes a 1-sphere $S\left(\sigma_{1}\right)$ that is contracted to the base point by $\tilde{h}_{i, 1, \sigma^{1}}$. Now consider the boundary of a 2-simplex $\sigma^{2} \subset \sigma^{l}$. Its boundary consists of three 1-simplices that can be turned into 1-spheres by attaching the fixed paths connecting their endpoints with the base point. As the result of contraction of these 1-spheres by $\tilde{h}$ we obtain three 2-discs. If we attach these discs to $\sigma^{2}$ and to each other along the common boundary we will obtain a simplicial 2-sphere $S\left(\sigma^{2}\right)$. Now proceed by induction. Eventually we consider $l+1(l-1)$-dimensional simplices in the boundary of $\sigma^{l}$. Each of them can be turned into a simplicial sphere by adding simplicial discs obtained by contracting simplicial spheres obtained from its boundary by $\tilde{h}$ (and gluing these discs together along common parts of their boundary. For any two discs this common part of their boundary is generated by the homotopy $\tilde{h}$ that contracts the simplicial sphere obtained from the intersection of the $(l-1)$-simplices corresponding to the 
discs.) As the result we will obtain a simplicial sphere denoted $S\left(\sigma^{l}\right)$ and called a special simplicial sphere corresponding to $\sigma^{l}$. Observe that $S\left(\sigma^{l}\right)$ will lie in $\mathcal{K}_{i+2^{l-1}-1}$. (This follows from the definition of $\tilde{h}_{i, l-1, \sigma^{l-1}}$ that is supposed to contract special simplicial spheres obtained from $(l-1)$-dimensional simplices of $\mathcal{K}_{i}$ inside $\mathcal{K}_{i+2^{l-1}-1}$.) The homotopy $\tilde{h}_{i, l, \sigma^{l}}$ will be a homotopy that contracts $S\left(\sigma^{l}\right)$ in $\mathcal{K}_{i+2^{l}-1}$ defined in the proof of Lemma 5.1 below.

Now let us explain the construction of $\tilde{\tilde{g}}_{i, l}$. We will begin by considering $\tilde{\tilde{g}}_{i, 0}: \operatorname{Comp}_{i, 0} \longrightarrow \mathcal{K}_{i}$, where $\operatorname{Comp}_{i, 0}$ is obtained from $\mathcal{K}_{i}$ by identifying its 0 -skeleton with a point $\tilde{v}_{0}$. Let us construct $\tilde{\tilde{g}}_{i, 0}$ by extending it from skeleton to skeleton.

First, let us consider the 0 -skeleton of $\operatorname{Comp}_{i, 0}$. It consists of a single vertex $\tilde{\tilde{v}}_{0}$. Naturally, we will let the image of it be equal to $\tilde{v}_{0}$.

Next, the 1-skeleton of $\operatorname{Comp}_{i, 0}$ will consist of a wedge of 1-spheres, with a sphere for each simplex of $\mathcal{K}_{i}$. Consider a typical sphere $\tilde{\tilde{S}}^{1}$ that corresponds to the edge $\tilde{e}$ that joins both vertices $\tilde{v}_{j}$ and $\tilde{v}_{l}$. Both of those vertices can be joined with $\tilde{v}_{0}$ by minimal simplicial paths: $\tilde{P}_{j}$ and $\tilde{P}_{l}$ respectively. Then $\tilde{P}_{j} \cup \tilde{e} \cup-\tilde{P}_{l}$ is a simplicial 1-sphere. We will let the image of $\tilde{\tilde{S}}^{1}$ under $\tilde{\tilde{g}}_{i, 0}$ be that sphere.

Now, we will extend this map to the 2-skeleton, which consists of 2-cells: a cell for each 2-simplex of $\mathcal{K}_{i}$. Each such cell will be mapped to the corresponding simplex together with one dimensional paths "attached" to each vertex of this simplex, each taken twice in different directions.

Similarly, we can extend this map to any skeleton of the complex, the image of an $m$-cell being a corresponding $m$-simplex in $\mathcal{K}_{i}$ together with paths that connect each vertex to $\tilde{v}_{0}$ taken twice, but with different orientations.

Now we will consider the general case of a complex Comp $i, k$. Its 0 -skeleton will always consist of a single vertex $\tilde{\tilde{v}}_{0}$, which will always be mapped to a vertex $\tilde{v}_{0}$ of $\mathcal{K}_{i}$. It will not have any cells in dimensions between 0 and $(k+1)$. Consider its $(k+1)$-skeleton. It will be a wedge of spheres of dimension $(k+1)$ : a sphere for each $(k+1)$-simplex of $\mathcal{K}_{i}$. We will want to know where to map a typical sphere. Consider the corresponding simplex $\tilde{\sigma}^{k+1}=\left[\tilde{v}_{1}, \ldots \tilde{v}_{k+2}\right]$ of $\mathcal{K}_{i}$. Using homotopies $\tilde{h}$ we can construct the special simplicial sphere $S\left(\tilde{\sigma}^{k+1}\right) \in \mathcal{K}_{i+2^{k}-1}$ as above. The cell corresponding to $\tilde{\sigma}^{k+1}$ will be mapped into this simplicial sphere. (The image of $\tilde{\sigma}^{k+1}$ minus an annulus near its boundary in $\operatorname{Comp}_{i, k}$ will be mapped into the image of $\tilde{\sigma}^{k+1}$ in $\mathcal{K}_{i+2^{k}-1}$ whenever the image of the annulus near the boundary of $\tilde{\sigma}^{k+1}$ in $\operatorname{Comp}_{i, k}$ will be mapped using the homotopy contracting the boundary of $\tilde{\sigma}^{k+1}$ to the base point in $\mathcal{K}_{i+2^{k}-1}$ made of the homotopies $\tilde{h}$.)

To extend this homotopy to higher skeleta we proceed similarly to the case of $\operatorname{Comp}_{i, 0}$. For example, consider $(k+2)$-skeleton of Comp $p_{i, k}$ and consider the $(k+2)$-cell $\tilde{\tilde{\sigma}}^{k+2}$, the image of $\tilde{\sigma}^{k+2}$ under the quotient map. Let $\partial \tilde{\tilde{\sigma}}^{k+2}=\tilde{\tilde{S}}_{1}^{k+1}+$ $\ldots+\tilde{\tilde{S}}_{k+3}^{k+1}$. Subdivide this cell into the inner disc and the outside annulus. Map the inner disc onto the $\tilde{\sigma}^{k+2}$. The outer boundary of the annulus is mapped using $k+3$ maps of cells of Comp $_{i, k}$ constructed in the previous step. The annulus is mapped using a homotopy between the maps of its inner and outer boundaries. 
This homotopy is just the extension of the homotopies $\tilde{h}$ contracting boundaries of $(k+1)$-dimensional cells $\tilde{\sigma}^{k+1}$ in the boundary of $\tilde{\sigma}^{k+2}$ using the standard extension technique "wagon in dirt".

Once again note, that in order to construct maps from $\operatorname{Comp}_{i, k}$ to $\mathcal{K}_{i-1+2^{k}}$ and to $M^{n}$ we fixed homotopies $\tilde{h}$ that connect special simplicial spheres with a point. In Sect. 5 we will construct $\tilde{h}$ with following properties:

1. The simplicial disc $\tilde{D}^{k+1}$ generated by this homotopy will have a controllable number of simplices, (i.e. the number of simplices can be bounded from above in terms of the number of simplices of $\mathcal{K}_{j}$ or in terms of the available parameters of the manifold);

2. $h_{i, k, \sigma^{k}}=g_{i-1+2^{k}} \circ \tilde{h}_{i, k, \sigma^{k}}$ will have a controlable width.

Now recall that $\mathcal{K}_{1}$ is the $(n+1)$-dimensional skeleton of the nerve of a minimal $\delta$-net in $M^{n}$, that $n_{0}>1$ denotes the number of vertices, i.e. points in the net, that $R$ is the lower bound for the contractibility radius of $M^{n}$ (in the case when $M^{n}$ satisfies conditions of Theorem 0.1 we define $R=\pi$ ), and that $\delta_{i}<R$ are radii of balls of the covers of $M^{n}$ used to construct $\mathcal{K}_{i}$. The main goal of the next two and a half sections will be to present an inductive proof the following proposition:

Proposition 3.2 (The width of homotopies). Let $M^{n}$ be a closed $(q-1)$ connected Riemannian manifold satisfying the assumptions of Theorem 0.1 or Theorem 0.2. Assume that any closed curve in $M^{n}$ of length $\leq 3 n_{0} R$ can be contracted by a length non-increasing homotopy. Let $S^{k}$ be any simplicial sphere in $M^{n}$, (i.e. $S^{k}=g_{1}\left(\tilde{S}^{k}\right)$, where $\tilde{S}^{k}$ is a simplicial sphere in $\left.\mathcal{K}_{1}\right)$. Then there exists a homotopy $h_{\tau}^{k}$ that connects this sphere to $v_{0}$, such that in

Case A injrad $\left(M^{n}\right) \geq \pi$, the width of this homotopy is bounded from above by $(\delta+1) n_{0}^{\xi(n) n_{0}^{n}}$.

Case $\mathbf{B} M^{n} \in \mathcal{M}_{-1, v}^{d}$, the width of the homotopy is bounded from above by

$$
\left(C^{c_{1}(n)} \delta+1\right) \cdot n_{0}^{c(n) \cdot n_{0}^{n}} \text {. }
$$

Proposition 3.2 is a technical version of the following informal assertion: Let $M^{n}$ be a closed $(q-1)$-connected Riemannian manifold. Let $M^{n}$ be either triangulated into "small" simplices or endowed with a triangulation tower as above. Assume that any closed curve in $M^{n}$ of length less than a certain quantity $L\left(M^{n}\right)$ that can be explicitly determined can be contracted without increasing its length. Then each contractible simplicial sphere of dimension $\leq q$ can be contracted by a homotopy of controlled width. Observe that here we can take $L\left(M^{n}\right)=2 \operatorname{diam}\left(M^{n}\right)$, but we do not need this fact. Also, this assertion generalizes to the case when the sphere that we need to contract is not necessarily simplicial. This generalization will be stated as Theorem 5.2 and proven in Sect. 5. Finally, our proof of this informal assertion will work equally well if instead of assuming that closed curves of small length in $M^{n}$ can be contracted without increase of their length we will assume that the length during a contracting homotopy increases not more than by a factor $\alpha$. (Of course, $\alpha$ will then enter into the expresssion for the upper bound for the width of the contracting homotopy). 
In this section we will prove the first step of the induction.

Lemma 3.3 (First step). Let $\tilde{\gamma}: S^{1} \longrightarrow \mathcal{K}_{i}$ be a 1-dimensional simplicial sphere in $\mathcal{K}_{i}$, and $\gamma=g_{i} \circ \tilde{\gamma}: S^{1} \longrightarrow M^{n}$ be a piecewise geodesic curve of length $\leq 3 n_{0} \delta_{i}$ for some $i<2^{q}$. Assume that all closed curves in $M^{n}$ of length $\leq 3 n_{0} R$ can be contracted to a point by a length non-increasing homotopy. (Recall that $n_{0}$ is the number of vertices in $\mathcal{K}_{i}$. In Case $A 3 n_{0} R$ is majorized by $c(n) \operatorname{vol}\left(M^{n}\right)$.)

Then there exists a homotopy $h_{\tau}^{1}=g_{i+1} \circ \tilde{h}_{\tau}^{1}$ of $\gamma$ to a point, that has the following properties:

\section{Case A.}

1. The width of this homotopy $W_{h_{\tau}^{1}} \leq c_{1}(n) \cdot\left(\operatorname{vol} M^{n}\right)^{c_{2}(n) \cdot v^{\prime} M^{n}}$.

2. $\tilde{h}_{\tau}^{1}$ is a simplicial homotopy that connects $\tilde{\gamma}$ with a point in $\mathcal{K}_{i+1}$, and the number of 2-simplices forming this homotopy and counted with multiplicities is bounded from above by $c_{3}(n) \operatorname{vol}\left(M^{n}\right)^{c_{4}(n) \operatorname{vol}\left(M^{n}\right)}$.

\section{Case B.}

1. The width of this homotopy $W_{h_{\tau}^{1}} \leq n_{0}^{c(n) n_{0}}\left(C \delta+3 n_{0} R / \delta\right)$.

2. $\tilde{h}_{\tau}^{1}$ is a simplicial homotopy that connects $\tilde{\gamma}$ with a point in $\mathcal{K}_{i+1}$, and the number of 2-simplices of $\mathcal{K}_{i+1}$ forming this homotopy and counted with multiplicities is bounded from above by $n_{0}^{c_{1}(n) n_{0}}$.

Lemma 3.3 is almost a particular case of Proposition 3.2 when $k=1$ and the closed curve that we want to contract is short. (The assumption of shortness will be dropped in Lemma 3.4 below). But it asserts somewhat more. Namely, it asserts that the contracting homotopy of controlled width can be chosen to be the projection of a simplicial homotopy (in $\mathcal{K}_{i+1}$ ) with a controlled number of simplices.

Proof. The proof of this statement is very similar to the proof of Lemma 3.5(1) in [R]. Therefore here we will present an outline of the proof only.

We can first construct an $\varepsilon$-net in the neighborhood of the space of all closed curves in $M^{n}$ of length bounded from above by $3 n_{0} \delta_{i}$, where $\varepsilon=3 \delta_{i}$.

In fact, this net is constructed by considering a 1 -skeleton of $\mathcal{K}_{i}$, and by taking all subcomplexes that form closed curves, the length of which is bounded by $100 n_{0} \delta_{i}$.

Then, using the fact that our curve is contractible, one can construct a sequence of simplicial curves, that starts with our original curve and ends with a point and estimate the number of curves in this sequence, where the distance between two consecutive curves is smaller than $10 \delta_{i}$.

After that it only remains to show that two consecutive curves in the sequence can be joined with a thin homotopy and the proof will be completed.

The idea is to decompose their "difference" into small "triangles" (or "quadrangles") and to use the homotopies contracting these "triangles" in $\mathcal{K}_{i+1}$. (Each of these triangles bounds a 2 -simplex in the $(i+1)$ st nerve.) The details of how one can use the homotopies of these short "triangles" in order to construct the homotopy between the long curves can be found in [R]. 
The same trick as in the proof of Theorem 3.6 of [R] can be used to generalize this lemma to the situation when the length of $\gamma$ is not bounded. Namely one can choose any vertex and decompose any closed curve into a sum of closed curves of length $<3 d$ using the "spikes in a wheel" trick. This trick and the previous lemma immediately impy the following result:

Lemma 3.4. Let $M^{n}$ be a closed Riemannian manifold of dimension $n$. Assume that any closed curve of length $\leq 3 n_{0} \delta_{i}$ can be contracted to a point without length increase. Let $\gamma: S^{1} \longrightarrow M^{n}$ be a piecewise differentiable curve that consists of $Q$ piecewise geodesics between adjoint vertices of $\mathcal{K}_{i}$. Then there exists a homotopy that connects this curve to a point, and satisfies the same conditions as in the previous lemma with the only difference that the upper bounds for the number of 2-simplices of $\mathcal{K}_{i+1}$ in Case A.2 and Case B.2 becomes $c_{3}(n) Q \operatorname{vol}\left(M^{n}\right)^{c_{4}(n) \operatorname{vol}\left(M^{n}\right)}$ and $Q n_{0}^{c(n) n_{0}}$, correspondingly.

Proof. The proof is very similar to that of Lemma 3.6 in [R].

\section{Linear Algebra and homological "filling"}

In this section we find an upper bound for the number of simplices in the minimal chain that bounds a null homologous cycle by interpreting this question as a question from Linear Algebra. By "the number of simplices" in a chain $\sum_{i} n_{i} \sigma_{i}, n_{i} \in Z, \sigma_{i}$ is a simplex, we mean $\sum_{i}\left|n_{i}\right|$. Let us start from the following lemma from Linear Algebra:

Lemma 4.1 (Linear Algebra). Let $A$ be an $l \times m$ matrix with integer entries and $b=\left(b_{1}, \ldots, b_{l}\right) \in Z^{l}$ be an integer vector. Suppose also that the equation $A x=b$ has an integer solution $x \in Z^{m}$. Then it has an integer solution $x_{*}=\left(x_{1}, \ldots, x_{m}\right)$ such that

$$
\left|x_{i}\right| \leq l^{l+1} M_{A}^{2 l}\left(M_{b}+m\right), i=1, \ldots, m,
$$

where $M_{A}=\max _{i, j}\left|a_{i j}\right|, M_{b}=\max _{i}\left|b_{i}\right|$.

Proof. First note that the system is equivalent to a subsystem that consists of $r$ equations, where $r=\operatorname{rank}(A) \leq l$. Denote the $r \times m$ matrix of this new system by $A_{0}$ and the right hand side vector by $b_{0}$. Without any loss of generality we can assume that the $r \times r$ submatrix $A_{r}$ of $A_{0}$ formed by its first $r$ columns is invertible. Let $B_{r}$ denote the submatrix of $A_{r}$ formed by its remaining $m-r$ columns. Then the general real solution $\left(x_{1}, \ldots, x_{m}\right)$ of the system $A_{0} x=b_{0}$ and, thus, of the original system can be written as follows: $x_{r+1}, \ldots, x_{m}$ are arbitrary and the vector $x^{(r)}=\left(x_{1}, \ldots, x_{r}\right)=A_{r}^{-1}\left(b_{0}-B_{r} x^{(m-r)}\right)=\frac{1}{\operatorname{det} A_{r}}\left(\operatorname{adj} A_{r}\right)\left(b_{0}-B_{r} x^{(m-r)}\right)$. Here $x^{(m-r)}$ denotes the vector $\left(x_{r+1}, \ldots, x_{m}\right)$ and $\operatorname{adj} A$ denotes the adjoint matrix of $A_{r}$. If $x^{(m-r)}$ has integer entries then the solution will be integer if and only if every entry of $\operatorname{adj} A_{r}\left(b_{0}-B_{r} x^{(m-r)}\right)$ is divisible by $\operatorname{det} A_{r}$. But all entries of $\operatorname{adj} A_{r}\left(b_{0}-\right.$ $\left.B_{r} x^{(m-r)}\right)\left(\bmod \operatorname{det} A_{r}\right)$ are periodic with period $\operatorname{det} A_{r}$ with respect to each of the variables $x_{r+1}, \ldots, x_{m}$. So, if there is an integer solution of the system then there is an integer solution of the system with $x_{r+1}, \ldots, x_{m} \in\left\{0,1,2, \ldots, \mid\right.$ det $\left.A_{r} \mid-1\right\}$. 
Now it remains to notice that $1 \leq\left|\operatorname{det} A_{r}\right| \leq l^{\frac{l}{2}} M_{A}^{l}, \max _{i, j}\left(B_{r}\right)_{i j} \leq M_{A}$ and the absolute value of any entry of $\operatorname{adj} A_{r}$ does not exceed $l^{\frac{l}{2}} M_{A}^{l-1}$.

Corollary 1 (Number of simplices). Let $c_{k}=\sum_{i} a_{k}^{i} \sigma_{i}^{k}$ be a k-cycle in a simplicial complex with $n_{0}$ vertices that bounds a $(k+1)$-dimensional chain. Then this cycle bounds a $(k+1)$-dimensional chain $c_{k+1}=\sum a_{k+1}^{i} \sigma_{i}^{k+1}$, where $\sum_{i}\left|a_{k+1}^{i}\right| \leq$ $n_{0}^{4 n} n_{0}^{n} \max _{i}\left|a_{k}^{i}\right|$.

Proof. Let us apply Lemma 3.1 in the situation when $A$ is the matrix of the boundary operator $\partial_{k+1}$ from the chain complex $C_{k+1}$ freely generated by all $(k+1)$-dimensional simplices to the chain complex freely generated by all $k$ dimensional simplices. It is clear that $M_{A}=\max _{i j}\left|a_{i j}\right|=1, m=n_{k+1}, l=n_{k}$. Let $b$ be the vector of coefficients at all $k$-simplices of $c_{k}$. So, $M_{b}=\max _{i}\left|a_{k}^{i}\right|$. Vectors of coefficients of $(k+1)$-chains bounded by $c_{k}$ are integer solutions of the equation $A x=b$. Now we can apply Lemma 3.1. It remains to notice that for any $k \leq n n_{k} \leq\left(\begin{array}{c}n_{0} \\ k\end{array}\right) \leq n_{0}^{n}$.

\section{Proof of Proposition 3.2}

The goal of this section is to establish Proposition 3.2 and to extend it to the case where the sphere that we want to contract is not necessarily simplicial.

Assume that for some $N_{1}$ for any 1-dimensional simplicial sphere in $\mathcal{K}_{i}$ that is composed of not more than $3 n_{0}$ simplices there is a simplicial homotopy that connects this sphere with a point, and the disc generated by this homotopy is composed of no more than $N_{1}$ simplices. First, we are going to prove the following lemma under the assumption just made:

Lemma 5.1. Let $\tilde{S}^{k}$ be a simplicial sphere of dimension $k \leq(q-1)$ or a contractible simplicial sphere of dimension $q$ in $\mathcal{K}_{i}$ composed of $Q$ simplices. Then it can be contracted to a point $\tilde{v}_{0}$ along a simplicial disc $\tilde{D}^{k+1}$ in $\mathcal{K}_{i+2^{k-1}}$, which will be composed of no more than $c_{1}(n) Q n_{0}^{c_{2}(n) n_{0}^{n}} N_{1}$ simplices.

Proof. We know how to construct homotopies for a sphere of dimension 1 from our assumption.

Now assume that we have learned to construct such homotopies for spheres up to and including dimension $k-1$.

We want to construct a homotopy for any simplicial sphere $\tilde{S}^{k}$. The desired homotopy will be constructed in three steps.

Step 1. Let $\tilde{S}^{k}$ be a simplicial sphere, composed of $Q$ simplices. This sphere is contractible in $\mathcal{K}_{i+1}$, therefore, it bounds a chain in $\mathcal{K}_{i+1}$. Therefore, we can find an "optimal" such chain $\tilde{C}_{k+1}=\sum a_{k+1}^{j} \tilde{\sigma}_{j}^{k+1}$ that has $\tilde{S}_{k}$ as its boundary. The number of simplices in $\tilde{C}_{k+1}$ is bounded as in Corollary 1 .

Step 2. Consider $\operatorname{Comp}_{i+1, k-1}$ and the image $\tilde{\tilde{S}}^{k}$ of $\tilde{S}^{k}$ in it. $\tilde{\tilde{S}}^{k}$ will be a wedge of spheres, with one sphere corresponding to each of $k$-simplices that compose $\tilde{S}^{k}$. 
$\tilde{\tilde{S}}^{k}$ bounds the chain $\tilde{\tilde{C}}_{k+1}$, the image of $\tilde{C}_{k+1}$, therefore it is contractible to a point $\tilde{\tilde{v}}_{0}$. In this step we construct a contracting homotopy that will consist of homotopies that create $(k+1)$-cells of $\mathcal{K}_{i+1}$ as well as of some auxilliary homotopies discussed later.

The basic "building blocks" are homotopies that create cells and are the images in Comp $_{i+1, k-1}$ of homotopies creating the boundary of a $(k+1)$-simplex from a constant map (see Fig. 9).
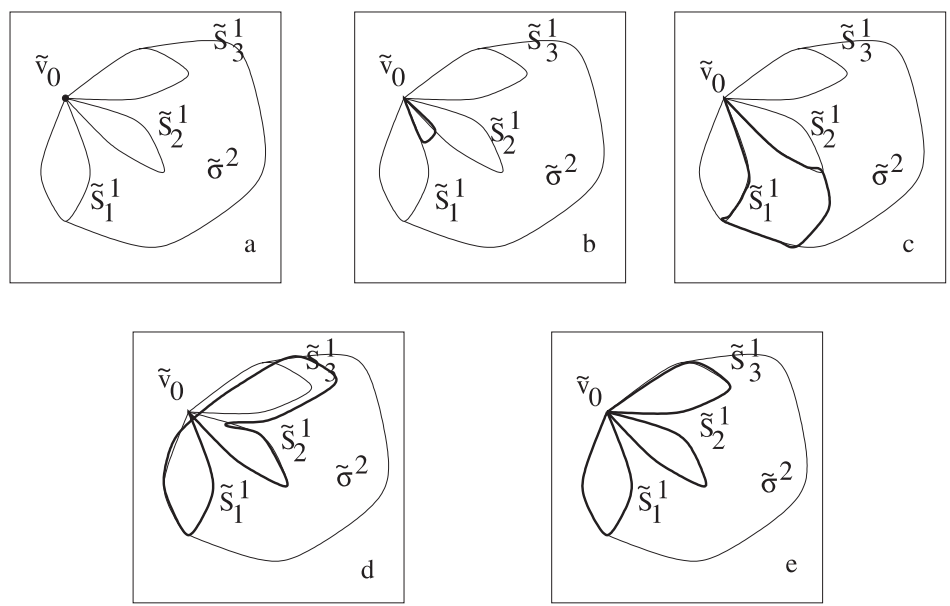

Fig. 9. A homotopy that connects a constant mapping with the boundary of the 2-cell

We will need $\sum_{i=1}^{n_{k+1}}\left|a_{k+1}^{i}\right|$ successive homotopies. Each of these successive homotopies adds (or subtracts) the boundary of a cell in Comp $_{i+1, k-1}$ which corresponds to a $(k+1)$-simplex in $\mathcal{K}_{i+1}$ (and therefore corresponds to a singular simplex in $M^{n}$ contained in a ball of radius much smaller than the contractibility radius of $M^{n}$. Later it will become important for us that as a conequence of this simplex being small the image of this homotopy in $M^{n}$ will have a small width.)

As the result we obtain a homotopy between $\tilde{\tilde{S}}^{k}$ and $\tilde{\tilde{S}}^{k}-\partial \sum_{i=1}^{n_{k+1}} a_{k+1}^{i} \tilde{\tilde{\sigma}}_{i}^{k+1}$. The last expression is equal to zero when regarded as a chain, and thus, it is homotopic to a point by a homotopy that is easy to construct.

The last homotopy can be explicitly described as follows: Let $\left\{\tilde{\tilde{S}}_{l}^{k}\right\}$ be spheres corresponding to each $k$-simplex in $\mathcal{K}_{i+1}$ Note that $\tilde{\tilde{S}}^{k}=\sum_{1}^{n_{k}} b_{l}^{k} \tilde{\tilde{S}}_{l}^{k}$, when regarded as a chain, and $\sum_{l=1}^{n_{k}} b_{k}^{l} \tilde{\tilde{S}}_{l}^{k}=\sum_{i=1}^{n_{k+1}} a_{k+1}^{i} \sum_{j=0}^{k+1} \tilde{\tilde{S}}_{i j}^{k}$, where $\tilde{\tilde{S}}_{i j}^{k}$ equals to $\pm \tilde{\tilde{S}}_{l}^{k}$ for some $l$, and $\sum_{j=0}^{k+1} \tilde{\tilde{S}}_{i j}^{k+1}$ corresponds to the image of the boundary of $\tilde{\sigma}_{i}^{k+1}$ in Comp $_{i+1, k-1}$. The fact that $\sum_{l=1}^{n_{k}} b_{k}^{l} \tilde{\tilde{S}}_{l}^{k}-\sum_{i=1}^{n_{k+1}} a_{k+1}^{i} \sum_{j=0}^{k+1} \tilde{\tilde{S}}_{i j}^{k}$ is equal to zero as a chain implies that each sphere $\tilde{\tilde{S}}_{l}^{k}$ in the above expression will be encountered 

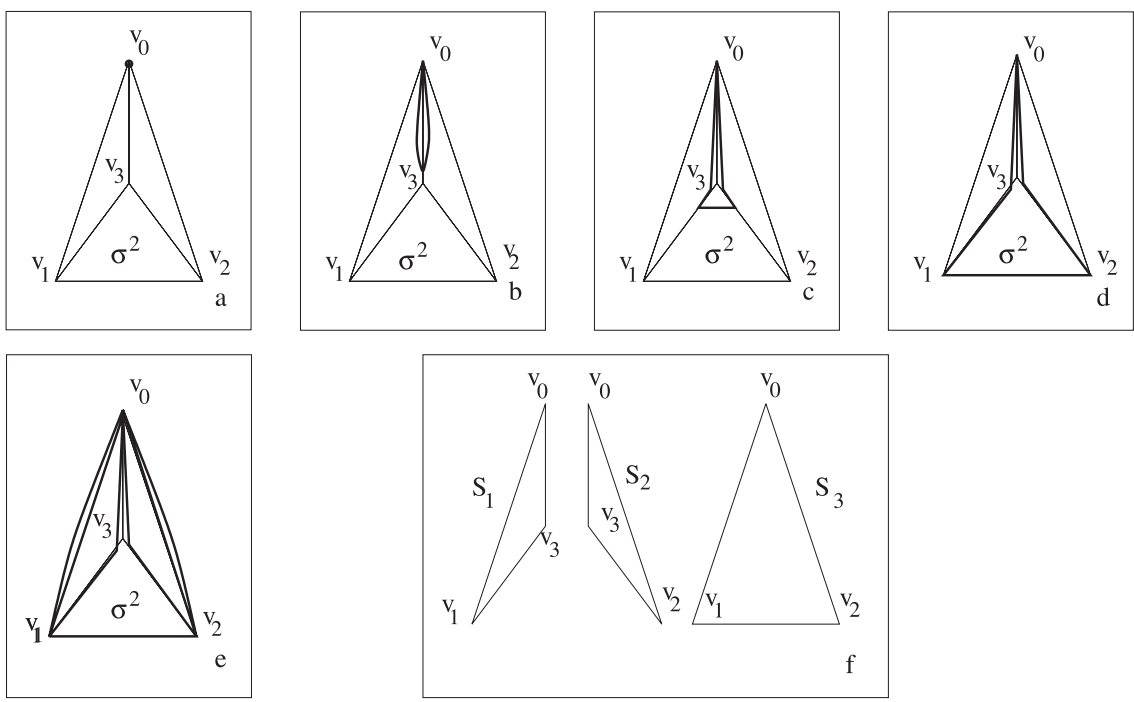

Fig. 10. How to construct a thin homotopy that connects a constant mapping with the "boundary" of a 2-cell in $M^{n}$

an even number of times, where the number of times it comes with a positive sign equals to the number of times it comes with a negative sign.

So, during the homotopy, we need to "move" the same spheres that come with a "+" sign and then with a "-" sign close to each other and then contract them to a point (by "move", we will mean a sequence of homotopies that exchange two spheres), see Fig. 14.

Step 3. Next consider $\tilde{\tilde{g}}_{i+1, k-1}:$ Comp $_{i+1, k-1} \longrightarrow \mathcal{K}_{i+2^{k-1}}$. Recall that the image of each closed cell of dimension $k$ (that is of a $k$-sphere) of Comp $_{i+1, k-1}$ is a simplex $\tilde{\sigma}^{k}=\left[\tilde{v}_{1}, \ldots, \tilde{v}_{k+1}\right]$ of dimension $k$ in $\mathcal{K}_{i+1}$ that corresponds to this sphere together with a disc that is generated by a homotopy of the boundary of this simplex that connects it with a point. More precisely, this homotopy of $\partial \tilde{\sigma}^{k}$ to $v_{0}$ is constructed as follows: Connect each vertex $\tilde{v}_{i}$ of this complex by a fixed path $\tilde{P}_{i}$, that lies in 1-skeleton of $\mathcal{K}_{i+1}$. Obtain 1-dimensional spheres $\tilde{P}_{i} \cup \tilde{e}_{i j} \cup-\tilde{P}_{j}$, where $\tilde{e}_{i j}$ is the edge joining $\tilde{v}_{i}$ and $\tilde{v}_{j}$. Those spheres can be contracted in 2-skeleton of $\mathcal{K}_{i+2}$. Next, consider 2 -spheres that are obtained from the 2-simplices in $\mathcal{K}_{i+1}$ and the disk obtained by contracting their boundary to the point. They lie in the 2-skeleton of $\mathcal{K}_{i+2}$. We can contract those spheres in the 3 -skeleton of $\mathcal{K}_{i+4}$. If we continue in the above manner we will reach the stage at which we will have to contract spheres of dimension $(k-1)$ that lie in $\mathcal{K}_{i+2^{k-1}}$ that are obtained from $\left[\tilde{v}_{1}, \ldots, \hat{\tilde{v}}_{j}, \ldots, \tilde{v}_{k+1}\right]$ togeher with the disk generated by the homotopy that connects the boundary of it with a point.

The image of the sphere $\tilde{\tilde{S}}^{k}$ under $\tilde{\tilde{g}}_{i+1, k-1}$ is contractible in $\mathcal{K}_{i+2^{k-1}}$, because $\tilde{\tilde{S}}^{k}$ is contractible in Comp $_{i+1, k-1}$. However, the image of the above sphere 
will not be our original sphere $\tilde{S}^{k}$. Let us denote the image sphere by $S^{\prime k}$. Recall that $\tilde{\tilde{g}}_{i+1, k-1}\left(\tilde{\tilde{f}}_{i+1, k-1}\left(\tilde{\sigma}^{k}\right)\right)$ is a simplex $\tilde{\sigma}^{k}$ together with the disc obtained by contracting its boundary to $\tilde{v}_{0}$. Note also that if $\tilde{\sigma}_{1}^{k}$ and $\tilde{\sigma}_{2}^{k}$ share a face then the homotopies that connect the boundary of each simplex with $\tilde{v}_{0}$ will agree when restricted to this face. Therefore, $S^{\prime k}$ will be $\tilde{S}^{k}$ with "inserted wedges" $\tilde{S F}_{j}^{k}$, where each $\tilde{S F}_{j}^{k}$ is a simplicial complex generated by a homotopy that connects each simplex of dimension $(k-1)$ of $\tilde{S}^{k}$ with the point $\tilde{v}_{0}$. (Note that this simplex together with the already fixed homotopy contracting its boundary is a $(k-1)$-sphere. By the induction hypothesis Lemma 5.1 is true for this sphere and $\tilde{S F}_{j}^{k}$ denotes the image of the contracting simplicial homotopy.) Note also that each $\tilde{S F}_{j}^{k}$ will enter twice with a different orientation and therefore can be easily cancelled by a homotopy. Therefore, we can see that $S^{\prime}$ is homotopic to $\tilde{S}^{k}$ in $\mathcal{K}_{i+2^{k-1}}$.

Important Remark 5.2. The proof of Lemma 5.1 provides us with homotopies $\tilde{h}_{i, l, \sigma^{l}}$ introduced at the beginning of Sect. 3 and used in the construction of the maps Comp $_{i, k} \longrightarrow \mathcal{K}_{i+2^{k}-1}$. We take the special simplicial sphere $S\left(\sigma^{l}\right) \subset \mathcal{K}_{i+2^{l-1}-1}$ and contract it in $\mathcal{K}_{i+2^{l-1}-1+2^{l-1}}$ as in the proof of Lemma 5.1. But a general simplicial sphere $\tilde{S}^{k}$ can be contracted in a somewhat different way. For each $k$-dimensional simplex $\sigma^{k}$ consider the corresponding special simplicial sphere $S\left(\sigma^{k}\right)$. (Recall that its construction involves contracting special simplicial spheres corresponding to lower dimensional simplices in the boundary of $\sigma^{k}$ using the homotopies $\tilde{h}$ constructed as in the proof of Lemma 5.1.) There is an obvious homotopy between $\tilde{S}^{k}$ and the union of these special simplicial spheres in $\mathcal{K}_{i+2^{k-1}-1}$ (see Figs. 11 (a)-(c) and 12 (A)-(C). This union consists of $\tilde{S}^{k}$ and surfaces obtained by contracting the special simplicial spheres corresponding to faces of $\sigma^{k}$. Each of these surfaces enters twice (or more generally an even number of times) with opposite orientations. The homotopy in question consists of inserting pairs of these surfaces.) So, it remains to contract each of the considered special simplicial spheres. Each of these special simplicial spheres is located in $\mathcal{K}_{i+2^{k-1}-1}$ and can be contracted as in the proof of Lemma 5.1 in $\mathcal{K}_{i+2^{k-1}-1+2^{k-1}}=\mathcal{K}_{i+2^{k}-1}$. Combining these homotopies we obtain a homotopy contracting $\tilde{S}^{k}$ (see Figs. 11 and 12). It is this homotopy that we will be using from now on. In order to explain its advantage over the homotopy of Lemma 5.1 observe that the number of simplices in any special simplicial sphere does not depend on the number of simplices of $\tilde{S}^{k}$. If $\mathcal{K}_{j}$ were metric spaces then the width of this homotopy also would be independent of $\tilde{S}^{k}$ and of the number of simplices in $\tilde{S}^{k}$. Therefore when we will construct a homotopy in $M^{n}$ using the simplicial homotopy in nerves introduced in the present remark we avoid the unpleasant dependence of the width of the homotopy on the number of simplices in intermediate simplicial spheres (see below for details).

Proof of Proposition 3.2. We must verify that the width of the pull-down to $M^{n}$ of the homotopy of a simplicial sphere $\tilde{S}^{k} \subset \mathcal{K}_{1}$ constructed as in Important Remark 5.2 is as stated in Proposition 3.2. It follows from the discussion in Important Remark 5.2 that it is sufficient to estimate the width of the pull-down to $M^{n}$ of the 

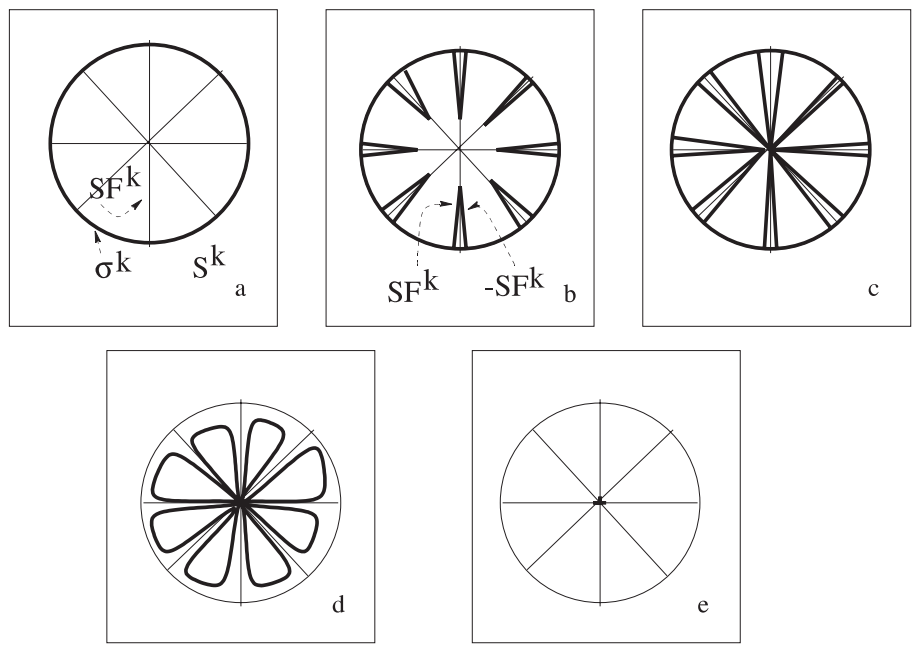

Fig. 11. The homotopy that connects $S^{k}$ with a point
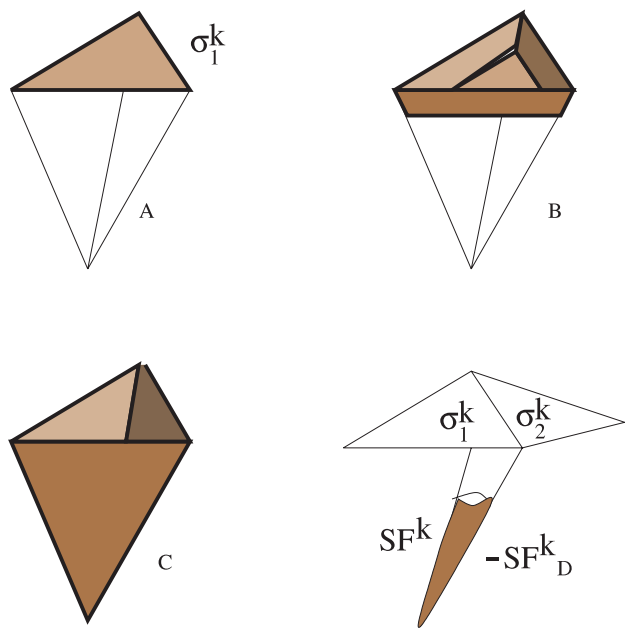

Fig. 12. The homotopy that connects $S^{k}$ with a point

homotopies of special simplicial spheres $\tilde{h}_{i, k, \sigma^{k}}$. We are going to do this by induction. The base of induction, $(k=1)$, is covered by Lemma 3.3. The homotopies $\tilde{h}$ can be broken into the following simpler homotopies:

1. The basic homotopy is the contraction of the boundary of a $k$-dimensional simplex through the simplex. The image of the boundary of a $k$-dimensional simplex from $\mathcal{K}_{j}$ in $M^{n}$ is contained in a metric ball of radius much less than the injectivity radius of $M^{n}$ (Case A) or the lower bound for the contractibility radius of $M^{n}$ 
(Case B). In fact when we introduced $g_{i}$ we mapped any simplex using a homotopy of controlled width contracting the already constructed map of its boundary to $M^{n}$ (see the proof of Lemma 2.3) (Recall that in Case A we homotoped along the geodesics connecting the image of the boundary with the image of one of the vertices. In Case B we used the homotopy of controlled width in a larger ball introduced by Grove and Petersen in [GrP].)

2. Permutation of two elements of $\pi_{k}\left(M^{n}\right)$ (see Fig. 14). A permutation of two elements of a homotopy group is illustrated on Fig. 14. Recall that in our situation each of these spheres corresponds to a standard simplicial sphere. We already know how to represent a $k$-dimensional short tentacled sphere as a $(k-1)$-dimensional sphere in the space of loops passing through the base point of controlled length. The standard simplicial sphere can be represented as the sphere in the space of loops of controlled length in exactly the same way. These loops can be used in order to move points while exchanging the $k$-spheres in $M^{n}$. However, we need the control over the length of two systems of loops going in different directions. Assume, for example, that we are permuting 2-dimensional spheres. Looking at the left side of Fig. 14 we see that we need a representation of each of these spheres by a map of the square $[0,1] \times[0,1]$ such that the boundary of the square is mapped to the point of intersection of two spheres and the images of straight line segments parallel to each side of the square have a controlled length. As we already observed we will have the required control over the length in one direction in our situation.

This control is due to the fact that the spheres we are considering are obtained by gluing two homotopies of bounded width of a closed curve of controlled length in the manifold. These homotopies are "made" out of homotopies of sufficiently close (less than the contractibility radius/5) closed curves. These last homotopies are "made" out of homotopies of bounded width of short curves obtained using the gradient flow of an appropriate vector field. (In the situation when $M^{n} \in \mathcal{M}_{-1, v}^{d}$ the existence of this field follows from ideas of Grove and Petersen; see [GrP].) It is precisely at this point that we loose the control over the length of curves that are being contracted during the homotopy because we do not have a control over the derivative of this vector field. (Whenever the width of the homotopy, i.e. the length of trajectories corresponds to one dimension of the square, the length of curves during homotopy corresponds to the second required dimension.) But one can use a trick from $[\mathrm{R}]$ in order to gain control over the length of curves during the homotopy (between two sufficiently close closed curves $\gamma_{1}$ and $\gamma_{2}$ ) without loosing control over the width. (See the proof of Proposition 5.1 in [R] and, in particular, Fig. 11 A-H in [R].) The trick can be called "an expanding tooth". We start from $\gamma_{1}$. First, we create an extremely narrow tooth between $\gamma_{1}$ and $\gamma_{2}$. Points of a very small interval in $\gamma_{1}$ go all the way to $\gamma_{2}$ along their trajectories (of bounded length); points outside a very small neighborhood of this interval stay on $\gamma_{1}$; points in between go along their respective trajectories but not all the way. Then we make this tooth wider and wider allowing points further and further from the initial narrow interval of $\gamma_{1}$ to reach $\gamma_{2}$. At any moment of time the image of $\gamma_{1}$ is made of a piece of $\gamma_{1}$, a piece of $\gamma_{2}$ and two curves that are extremely close to trajectories of two points of $\gamma_{1}$ under the homotopy (and therefore have length not exceeding the width of the homotopy plus an arbitrarily small number). 
So, we gained control over the length of the curves during the homotopy without loosing control over the width since each individual point will still move along its original trajectory. (Observe however that the Lipschitz constant for this homotopy will still be uncontrollably large!). Without any loss of generality we can assume that the homotopy between two sufficiently close closed curves in the proof of Lemma 3.3 is as described. It is clear that this trick immediately generalizes to higher dimensions giving us control over the length of the second system of loops required to permute two elements of the homotopy groups.

The number of these permutations does not exceed the square of the number of simplices of the standard simplicial sphere that is being contracted. This number obviously does not depend on the number of simplices in the original simplicial sphere that we are trying to contract.

3. Cancellation of pairs of constructed spheres with opposite orientations. Again we use the possibility to represent standard simplicial spheres by maps $[0,1]^{k-1} \times$ $[0,1] \longrightarrow M^{n}$ such that the boundary of the cube is mapped to the base point and the length of any circle obtained as the image of $\{x\} \times[0,1]$ is bounded in terms of the width of homotopies contracting spheres of dimension $k-1$ forming the tentacles of the sphere. This possibility was already discussed in Sect. 1 (for short-tentacled spheres). More details can be found in Sect. 6 (see the proofs of Theorems 0.1 and 0.2 there). The fact that the width of homotopies corresponding to this step is bounded by $2 \sup _{x}$ length(the image of $\left.x \times[0,1]\right)$ is now obvious.
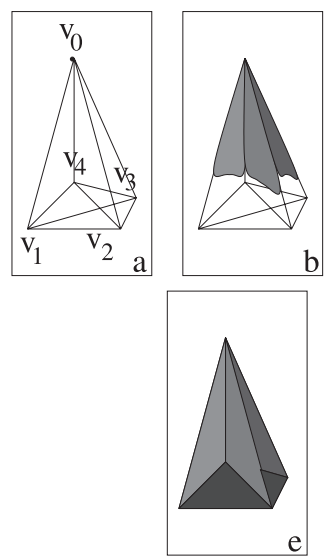
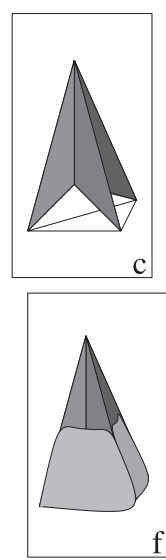
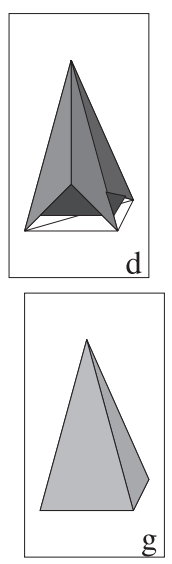

$g$

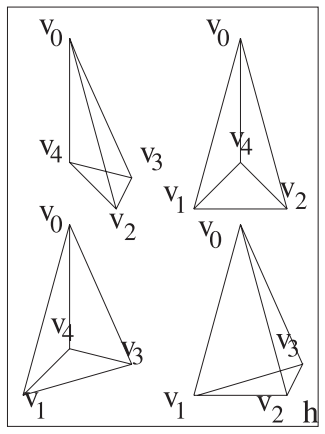

Fig. 13. How to construct a thin homotopy that connects a constant mapping with the mapping that takes a sphere to the "boundary" of a three dimensional cell in $M^{n}$

The above provides us with a recursive formula for the width of the homotopies and implies Proposition 3.2.

Next, we generalize Proposition 3.2 to the case of an arbitrary (not necessarily simplicial) contractible sphere: 


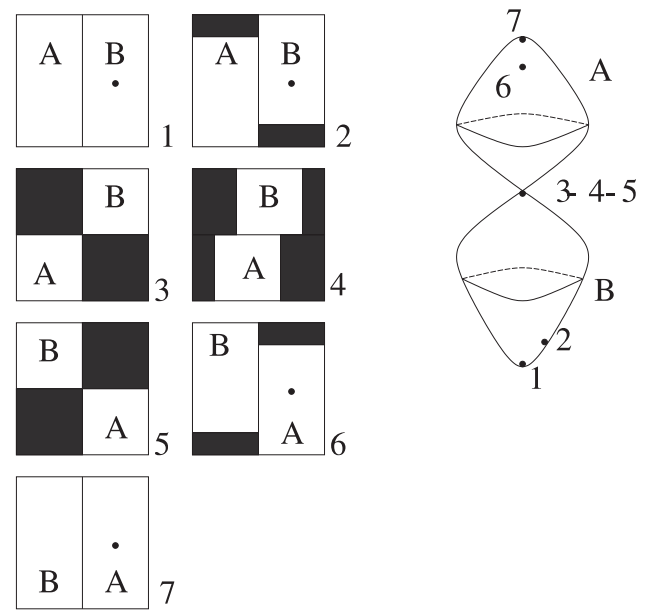

Fig. 14. A homotopy of exchanging two cells

Theorem 5.2. Let $M^{n}$ be a compact, $(q-1)$-connected Riemannian manifold and let

(1) $\operatorname{injrad}\left(M^{n}\right) \geq \pi$; or

(2) $M^{n} \in \mathcal{M}_{-1, v}^{d}$.

Assume that all closed curves in $M^{n}$ of length $\leq 3 n_{0} R$ can be contracted by a homotopy not increasing their length. (In Case (1) $R=\pi$, in Case (2) $R$ is the Grove-Petersen lower bound for the contractibility radius of $M^{n}$.)

Let $f: S^{k} \longrightarrow M^{n}$ be an arbitrary continuous map, where $1 \leq k \leq q-1$, or an arbitrary contractible map $f: S^{q} \longrightarrow M^{n}$. Then there exists a homotopy $h_{\tau}^{k}$ between $f$ and a constant map, such that the width of this homotopy is bounded from above by

(1) $W_{h_{\tau}^{k}} \leq c_{1}(n) V^{c_{2}(n) V^{n}}$.

or, correspondingly,

(2) $W_{h_{\tau}^{k}} \leq \xi_{1}(n)\left(C^{c_{3}(n)} R+1\right) n_{0}^{\xi_{2}(n) n_{0}^{n}}$.

Proof. Approximate $f\left(S^{k}\right)$ by a simplicial sphere $S^{\prime k}=g_{1}\left(\tilde{S}^{k}\right)$, where $\tilde{S}^{k}$ is the simplicial approximation to $f_{1}\left(f\left(S^{k}\right)\right)$ in the $k$ - skeleton of $\mathcal{K}_{1}$. A homotopy that connects $f\left(S^{k}\right)$ with a point is then constructed in two steps. In the first step one shows that $f\left(S^{k}\right)$ is homotopic to $S^{\prime k}$ so that the width of this homotopy is not too large, and in the second step one contracts $S^{\prime}$ to a point as in Important Remark 5.2 following Lemma 5.1. The second step was already described above so it remains to describe the first step. Observe that $f$ is close to the composition of $f$, the simplicial approximation $f_{1}$, and $g_{1}$, and the distance can be majorized in terms of $\delta$ and the contractibility constant $C$. Now apply the standard Grove-Petersen 
technique (cf. Corollary 6 on p. 500 in [P2]). This Corollary implies that when we chose a controllably small $\delta f\left(S^{k}\right)$ and $S^{\prime k}$ are homotopic. (Recall that $\delta$ was defined in Sect. 2 above. It is the radius of the balls of the covering $\mathcal{U}_{1}$ used to construct the first nerve, $\mathcal{K}_{1}$.) Corollary 6 in [P2] does not say anything about the width of the homotopy but this information easily follows from the proof. Roughly speaking the proof works as follows. We consider a fine triangulation of $S^{k}$ that is being mapped into $M^{n}$ by the two close continuous maps. We construct inductively the homotopies between restrictions of these maps to skeleta of the triangulation of higher and higher dimension. Each of these homotopies is made of homotopies contracting small spheres in $M^{n}$. These spheres are so small that they lie either inside of a ball of radius $<\operatorname{injrad}\left(M^{n}\right)$ (Case A) or less than the contractibility radius of $M^{n}$ (Case B). In the first case the bound on the width is obvious; in the second it follows from the construction of the homotopy in the paper by K. Grove and P. Petersen [Gr P]. (We need here not only the contractibility radius and the (linear) contractibility function of $M^{n}$ but all the assumptions on the geometry of $M^{n}: K \geq-1, \operatorname{vol}\left(M^{n}\right) \geq v>0$ and $\operatorname{diam}\left(M^{n}\right) \leq d$.) We will spare the reader the details of the calculation.

\section{Proof of Theorems 0.1 and 0.2}

In this section we complete the proof of Theorems 0.1 and 0.2 .

Lemma 6.1. Let $M^{n}$ be a compact $(q-1)$-connected Riemannian manifold that is not q-connected. Assume that either injrad $\left(M^{n}\right) \geq \pi$ or $M^{n} \in \mathcal{M}_{-1, v}^{d}$. In the first case let $R=\pi$, in the second case let $R$ denote the Grove-Petersen lower bound for the contractibility radius of $M^{n}$. Assume that for any closed curve of length $\leq 3 n_{0} R$ there exists a homotopy that connects this curve with a point without length increase. Then there exists at least one short-tentacled sphere of dimension $q$ representing a non-trivial element of $\pi_{q}\left(M^{n}\right)$, such that the size of its tentacles is

$$
\begin{aligned}
& \leq c_{1}(n)\left(V^{c_{2}(n)\left(V^{n}+1\right)}+1\right) \quad \text { if injrad } \geq \pi . \\
& \leq \exp \left(\frac{\exp \left(c_{3}(n) d\right)}{\min \{1, v\}^{c_{4}(n)}}\right), \quad \text { if } M^{n} \in \mathcal{M}_{-1, v}^{d} .
\end{aligned}
$$

Proof. We will obtain this sphere as an obstruction to extension of an arbitrary noncontractible sphere $g: S^{q} \longrightarrow M^{n}$ to the disc $D^{q+1}$ as explained in Sect. 1.1(i) using homotopies provided by Theorem 5.2 to contract spheres of dimension $<q$ during the extension process.

Let $g: S^{q} \longrightarrow M^{n}$ represent any non-trivial element of $\pi_{q}\left(M^{n}\right)$. We are going to try to extend $g$ to $D^{q+1}$ that fills $S^{q}$. As an obstruction we will obtain a different non-contractible sphere on $M^{n}$. Since this sphere is obtained as a result of an explicit construction, we will have the desired control over its parameters.

Here is the procedure that we will use in order to extend $g$ inductively to skeleta of $D^{q+1}$. We will begin by considering a very fine triangulation of $S^{q}$ and extending it to the triangulation of $D^{q+1}$ as a cone over the triangulation of $S^{q}$. 
(This triangulation has one extra vertex, $p$, that can be thought of as the center of $D^{q+1}$ or as the vertex of the cone.)

Then we extend the map $g$ from $S^{q}$ to $D^{q+1}$ inductively extending this map to skeleta of higher and higher dimension. At each step but the last one it will be necessary to extend the map of $S^{i}$ to $M^{n}$ to a map of $D^{i+1}$, where $i \leq q-1$. We will be using homotopies of controlled width provided by Theorem 5.2. As a result boundaries of all simplices in the triangulation of $D^{q+1}$ will be mapped to short-tentacled spheres.

Now, note the following: if we are able to extend the map $g$ to the $(q+1)$ skeleton then we can extend it to the disk. We know that this is impossible, so at the last $(q+1)$-st stage this process should be interrupted. Therefore, there should exist a non-trivial short-tentacled sphere of dimension $q$.

Proof of Theorems 0.1 and 0.2 . If there exists a closed curve of length $\leq 3 n_{0} R$ that cannot be contracted without the increase of its length then there exists a closed geodesic of length $\leq 3 n_{0} R$ and both theorems immediately follow. So, the assumptions of Lemma 6.1 are satisfied and there exists a non-contractible short-tentacled $q$-dimensional sphere.

Now we are going to construct a map $F:[0,1] \longrightarrow \operatorname{Map}\left(S^{q-1}, M^{n}\right)$, where $F(t)=S_{t}^{q-1}(p)$, such that

(a) $F(0)=F(1)=$ a constant map;

(b) the length of each curve $S_{t}^{q-1}\left(p^{*}\right), t \in[0,1]$ is bounded from above by $L=c(n)\left(V^{c(n)\left(V^{n}+1\right)}+1\right)$, in the case of Theorem A, and it is bounded by $L=\exp \left(\frac{c_{1}(n) \exp \left(c_{2}(n) d\right)}{\min \{1, v\}^{c_{3}(n)}}\right)$ in the case of Theorem B.

It will remain to reinterpret this map as a map $\tilde{f}: S^{q-1} \longrightarrow \Lambda_{L} M^{n}$, as was explained in Sect. 1.1(i) (see also Fig. 6 above).

Then $\tilde{f}$ is not contractible, and $L$ will be our upper bound.

We will begin with a non-contractible short-tentacled sphere $f: S^{q}=\partial \sigma^{q+1} \rightarrow M^{n}$ constructed as in Lemma 6.1, (see Fig. 15) where $\sigma^{q+1}=\left[v_{1}, \ldots, v_{q+2}\right]$. By abuse of notation we will denote the image of $\partial \sigma^{q+1}$ also as $\partial \sigma^{q+1}$. $\partial \sigma^{q+1}=$ $\sum_{i=1}^{q+2}(-1)^{i-1}\left[v_{1}, \ldots, \hat{v}_{i}, \ldots, v_{q+2}\right]=\sigma_{1}^{q}-\sigma_{2}^{q}+\ldots+(-1)^{q-1} \sigma_{q+2}^{q}$. Each face $\left[v_{1}, \ldots, \hat{v}_{j}, \ldots, v_{q+2}\right]$ is mapped to the surface generated by contracting its boundary to a point by a homotopy of controlled width constructed above.

Let us consider $\sigma_{1}^{q}$, one of the proper faces of $\sigma^{q+1}$ of dimension $q$. The image of $\sigma_{1}^{q}$ is $S F_{1}^{q}$, which is generated by the homotopy that connects $f\left(\partial \sigma_{1}^{q}\right)$ with a point. Let us denote this homotopy by $H_{\tau}^{q-1} \cdot H_{0}^{q-1}\left(S^{q-1}\right)=f\left(\partial \sigma_{1}^{q}\right) \cdot H_{1}^{q-1}\left(S^{q}\right)=p$.

Let $\tilde{H}_{\tau}^{q-1}=H_{1-\tau}^{q-1}$, so the widths of $\tilde{H}_{\tau}^{q-1}$ and $H_{\tau}^{q-1}$ are the same and $\tilde{H}_{0}^{q-1}\left(S^{q-1}\right)=p ; \tilde{H}_{1}^{q-1}=f\left(\partial \sigma_{1}^{q}\right)$, (see Figs. $\left.16(\mathrm{c}, \mathrm{d}, \mathrm{e})\right)$. Note that the width of this homotopy is the same as the size of the tentacle, i.e. it is the same as the width of contracting the sphere of dimension $(q-1)$ to the point, provided by Proposition 3.2.

Next, consider the remaining $\sigma_{2}^{q}, \ldots, \sigma_{q+2}^{q}$ and let $\sigma_{i}^{q-1}=\sigma_{1}^{q} \cap \sigma_{i}^{q}$. The image of $\sigma_{i}^{q}$ is $S F_{i}^{q}$ and is obtained by contracting $\partial \sigma_{i}^{q}$ to a point. We will denote these homotopies $h_{\tau}^{q-1, i}$. 


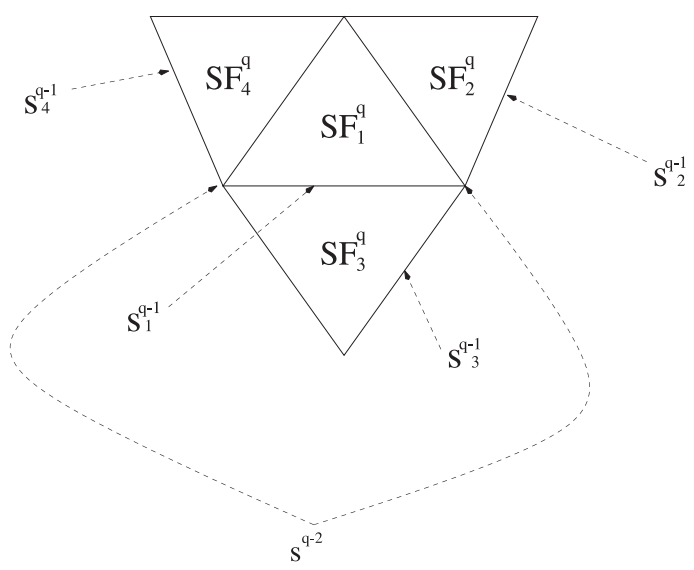

Fig. 15. A short-tentacled sphere $f: S^{q}=\partial \sigma^{q+1} \longrightarrow M^{n}$ "split" up
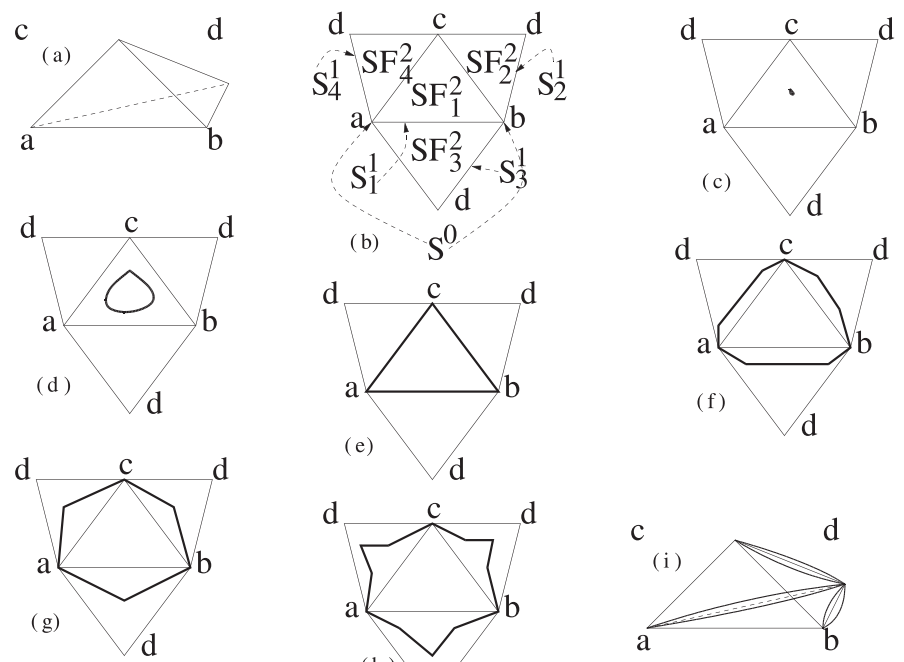

(b) ${ }^{\prime} \mathrm{S}^{0}$
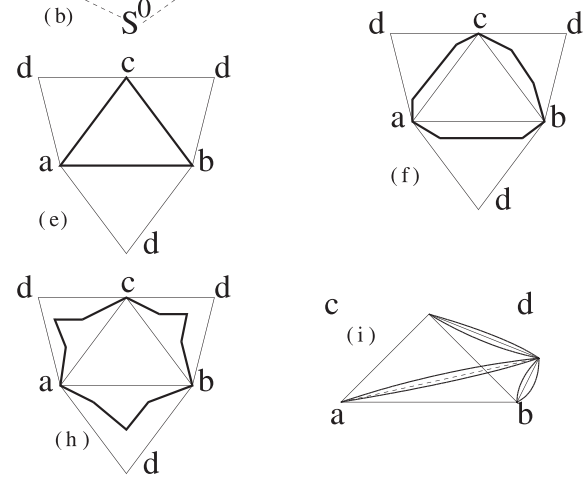

Fig. 16. Decomposing $S^{2}$ into $S^{1}$,s

Then there is a homotopy parametrized by $\tau^{*}$ that joins $\partial \sigma_{1}^{q}$ and the following $(q-1)$-dimensional sphere $S_{\tau^{*}}^{q-1}$ : instead of $\sigma_{i}^{q-1}$ consider the disc $D s_{i}^{q-1}$ that has the same boundary as $\sigma_{i}^{q-1}$ and is obtained by taking the annulus generated by $\left.h_{\tau}^{q-1, i}\right|_{\tau \in\left[0, \tau^{*}\right]}\left(\partial \sigma_{i}^{q-1}\right)$ together with the disc $h_{\tau^{*}}^{q-1, i}\left(\sigma_{i}^{q-1}\right)$. All discs $D s_{i}^{q-1}$ can be naturally glued along their boundaries. $S_{\tau^{*}}^{q-1}$ is the result of this glueing (see Figs. 16 (f,g), 17 (b)).

Now, consider a disc $\tilde{D} s_{i}^{q-1}$ that has the same boundary as before and is obtained by glueing the annulus $\left.h_{\tau}^{q-1, i}\right|_{\tau \in[0,1-\tilde{\tau}]}\left(\partial \sigma_{i}^{q-1}\right)$ and a disc that is obtained 
as $h_{1-\tilde{\tau}}^{q-1, i}\left(\partial \sigma_{i}^{q}-\sigma_{i}^{q-1}\right), \tilde{\tau} \in[0,1]$. These discs can be used to construct the obvious homotopy parametrized by $\tilde{\tau}$ (see Figs. 16(h), 17(d)).

Now we combine the above described three homotopies parametrized correspondingly by $\tau, \tau^{*}, \tilde{\tau}$ and obtain a homotopy between a point and a $(q-1)$-dimensional sphere $\left(\partial \sigma_{2}^{q}-\sigma_{2}^{q-1}\right) \cup \ldots \cup\left(\partial \sigma_{q+2}^{q}-\sigma_{q+2}^{q-1}\right)$, (see Figs. $\left.16(\mathrm{~g}, \mathrm{~h}), 17(\mathrm{~d}, \mathrm{e})\right)$. This $(q-1)$-sphere can be contracted to the vertex $v_{q+2}$ by "cancelling" simplices (= tentacles) $\sigma_{i j}^{q-1}=\partial \sigma_{i}^{q} \cap \partial \sigma_{j}^{q}, i, j \in\{2, \ldots,(q+2)\}$, each of which occurs twice with opposite orientation (see Fig. 16 (i)).
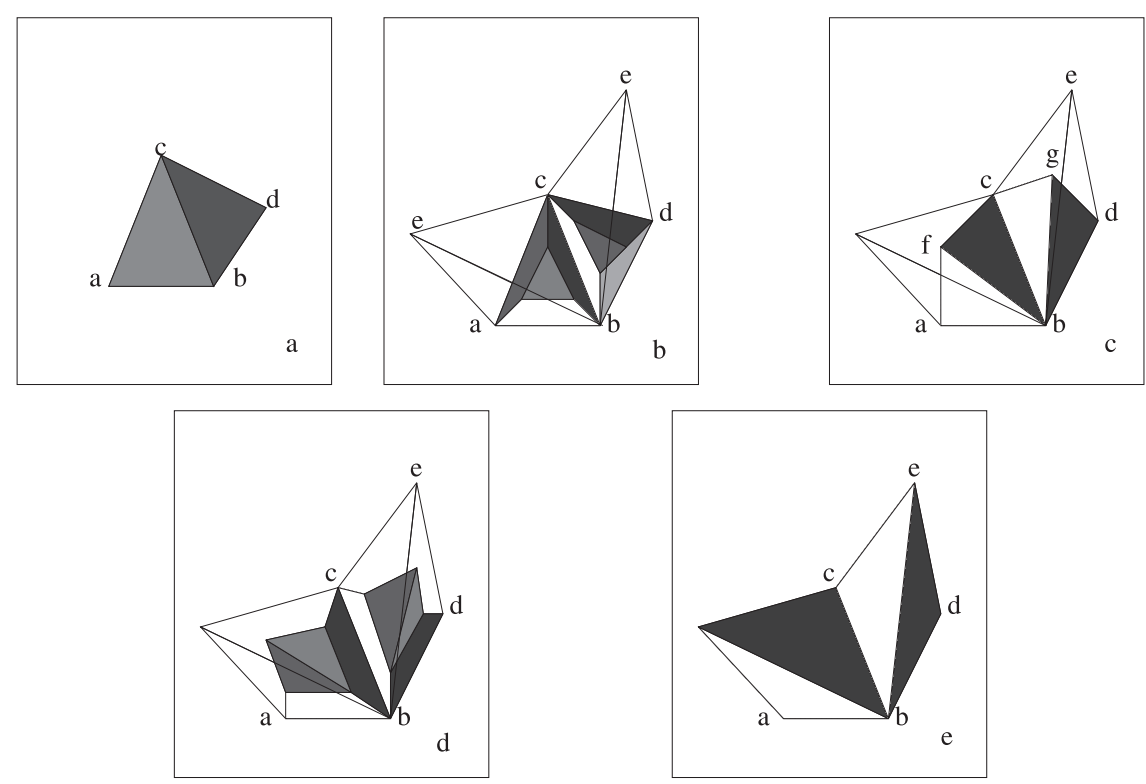

Fig. 17. "Decomposing" $S^{3}$ into spheres of smaller dimension

\section{Quantitative versions of the Hurewicz theorem}

Observe that Theorems $0.3,0.4$ are in fact already proven. They easily follow from Theorem 5.2 and Lemma 6.1. The only formal complication is that homotopies that connect short closed curves to a point can now increase their length by the factor of $\alpha$. But this just leads to the necessity of constructing nets in the space of longer curves in the Lemma 3.3. As the result one gets the $\alpha$ factor in Lemma 3.3 that propagates through all subsequent results.

In order to prove Theorem 0.5 we start from the (known) observation that the standard construction of a finitely presented group with unsolvable word problem as in [Rt] being combined with the version of the "witness" construction as in [Mil] yields an explicit infinite sequence of finite presentation of groups $G_{i}$ and two-dimensional finite simplicial complexes $K_{i}$ such that: 
1) For each of these groups $G_{i}$ either $G_{i}$ is trivial or $K_{i}$ is an aspherical CWcomplex with the fundamental group $G, K(G, 1)$;

2) There is no algorithm (= a Turing machine; or a computer program) that decides for a given $i$ whether or not $G_{i}$ is trivial;

3) For any $i \mathrm{H}_{2}\left(K_{i}\right) \neq 0$.

Here is a brief explanation how one can construct $G_{i}$ and $K_{i}$ : Theorem 4.12 in [Mil] asserts that there exists a finitely presented group $G$ with unsolvable word problem that can be obtained from a free group by applying three successive HNNextensions where the associated subgroups are finitely generated free groups. It is well-known that the classifying space of a free group is a wedge of circles. Also, the classifying space of an $\mathrm{HNN}$-extension $A_{* B}$ of a group $A$ with associated group $B$ can be described as $K(A, 1) \bigcup_{K(B, 1) \times\{0,1\}} K(B, 1) \times[0,1]$. Therefore $K(G, 1)$ can be represented by an explicit finite 2 -dimensional simplicial complex.

The witness construction described on p. 14 of [Mil] assigns to $G$ and any word $w$ in $G$ an explicit finite presentation of a group $G_{w}$ so that 1) $G_{w}$ is trivial if and only if $w=e$ in $G$; 2) If $w$ is non-trivial in $G$ then $G_{w}$ is an amalgamated free product of $G * Z$ and the free group with two generators over a free group. Therefore we obtain an explicit 2-dimensional finite complex $K_{w}$ such that if $w \neq 0$ in $G$, then $K_{w}=K\left(G_{w}, 1\right)$. It is not difficult to see that $K_{w}$ is the standard realization complex of the considered finite presentation of $G_{w}$ : it has one 1-cell corresponding to each generator and one 2-cell corresponding to each relator of the considered finite presentation of $G_{w}$. Examining relations of $G_{w}$ on p. 14 of [Mil] we see that $H_{2}\left(K_{w}\right) \neq 0$ for any $w$. Now we can enumerate all words $w$ in $G$ and define the groups $G_{i}$ and complexes $K_{i}$ as $G_{w}$ and $K_{w}$.

It is well-known that one can embed any finite two-dimensional simplicial complex $P$ into $R^{n+1}$ for any $n \geq 4$, consider the boundary of a small neighborhood of the embedded complex in $R^{n+1}$, and then smooth out corners of this boundary obtaining as the result a smooth hypersurface $M_{P}^{n}$ such that its fundamental group is isomorphic to $\pi_{1}(P)$ (see [BHP]). This hypersurface can then be triangulated. It is well-known that the whole construction of the triangulated $M_{P}^{n}$ from $P$ is effective (i.e. can be done by means of an algorithm). Further, it is easy to see that if $n>4$ then $\pi_{2}\left(M_{P}^{n}\right)$ also coincides with the second homotopy group of $P$ ([BHP]). Applying this construction to $K_{i}$ we obtain a sequence of manifolds $M_{i}^{n}$ such that $\pi_{1}\left(M_{i}^{n}\right)=G_{i}$ and $\pi_{2}\left(M_{i}^{n}\right)=\pi_{2}\left(K_{i}\right)=0$, if $G_{i}$ is non-trivial, and $\pi_{2}\left(M_{i}^{n}\right)=\pi_{2}\left(K_{i}\right)=H_{2}\left(K_{i}\right) \neq 0$, if $G_{i}$ is trivial. Therefore there is no algorithm deciding whether or not $\pi_{2}\left(M_{i}^{n}\right)$ is trivial.

Now let us prove Theorem 0.5 by contradiction. Assume that there exists a computable $f_{n}(N)$ as in the text of Theorem 0.5. We are going to construct an algorithm that decides the triviality of $\pi_{2}\left(M_{i}^{n}\right)$ and therefore obtain a contradiction as follows. It is well-known that there exists an algorithm listing for any $K$ the list of all triangulations of $S^{2}$ with $\leq K$ simplices. (Just list all simplicial 2complexes with $\leq K$ simplices and check which of them is a triangulation of $S^{2}$ ). So, compute $f_{n}(N)$ and construct the list of all triangulations of $S^{2}$ with $\leq f_{n}(N)$ simplices. For each of these triangulations there is a finite number of simplicial maps of this triangulation into the triangulation of $M_{i}^{n}$. All these simplicial maps 
can be listed. If $M_{i}^{n}$ is simply connected, then it has a non-trivial $\pi_{2}$, and one of these maps is non-contractible and therefore homologically non-trivial (by virtue of the Hurewicz theorem). If $M_{i}^{n}$ is non-simply connected, then it has trivial second homotopy group, and all these maps are homotopic to the constant map and, therefore, are homologically trivial. So, it is sufficient to check whether or not all these maps induce the trivial homomorphism between the second homology groups. But it is easy to verify by means of an algorithm whether or not a given simplicial map of $S^{2}$ into a simplicial complex sends the fundamental homology class of $S^{2}$ to zero. So, there is an algorithm telling us whether or not there is a homotopy non-trivial simplicial map of $S^{2}$ triangulated with $\leq f_{n}(N)$ simplices into $M_{i}^{n}$ which is equivalent to the non-vanishing of $\pi_{2}\left(M_{i}^{n}\right)$. The resulting contradiction completes the proof of Theorem 0.5 .

Acknowledgements. We would like to thank an anonymous referee of this paper for his/her comments that helped to improve its exposition.

Both authors acknowledge a partial support of this work by NSERC Research Grants. Regina Rotman acknowledges a partial support of this work by NSF Post-Doctoral Fellowship.

\section{References}

[B] Bott, R.: Lectures on Morse theory, old and new. Bull. Am. Math. Soc., New Ser. 7, 331-358 (1982)

[BalThZ] Ballmann, W., Thorbergsson, G., Ziller, W.: Existence of closed geodesics on positively curved manifolds. J. Differ. Geom. 18, 221-252 (1993)

[Br] Berger, M.: Systoles et applications selon Gromov. Seminaire Bourbaki, Vol. 1992/93. Astérisque 216, 279-310 (1993)

[BHP] Boone, W., Haken, W., Poénaru, V.: On recursively unsolvable problems in topology and their classification. In: Contributions to mathematical logic, ed. by H. Arnold Schmidt, K. Schutte, H.-J. Thiele. North-Holland 1968

[Ch] Chavel, I.: Riemannian geometry - a modern introduction. Cambridge: Cambridge University Press 1993

[Cg] Cheeger, J.: Finiteness theorems for Riemannian manifolds. Am. J. Math. 92, 61-74 (1970)

[Cr1] Croke, C.B.: Some isoperimetric inequalities and eigenvalue estimates. Ann. Sci. Éc. Norm. Supér., IV. Sér. 13, 419-435 (1980)

[Cr2] Croke, C.B.: Area and the length of the shortest closed geodesic. J. Differ. Geom. 27, 1-21 (1988)

[F] Fet, A.I.: Variational problems on closed manifolds. Mat. Sb. 30, 271-316 (1952)

[GoHL] Gallot, S., Hulin, D., Lafontaine, J.: Riemannian Geometry. Berlin-Heidelberg: Springer 1987

[G1] Gromov, M.: Homotopical effects of dilatation. J. Differ. Geom. 13, 303-310 (1978)

[G2] Gromov, M.: Filling Riemannian Manifolds. J. Differ. Geom. 18, 1-147 (1983)

[G3] Gromov, M.: Metric structures for Riemannian and Non-riemannian spaces. Birkhäuser 1999

[G4] Gromov, M.: Quantitative homotopy theory. In: Prospects in Mathematics. Princeton: Princeton University Press 2000

[GrP] Grove, K., Petersen, P.V.: Bounding homotopy types by geometry. Ann. Math. 128, 195-206 (1988) 
[M] Maeda, M.: The length of a closed geodesic on a compact surface. Kyushu J. Math. 48, 9-18 (1994)

[Mil] Miller, C.: Decision problems for groups - survey and reflections. In: Combinatorial group theory, ed. by G. Baumslag and C. Miller, 1-59. Springer 1989

[M1] Milnor, J.: Morse Theory. Princeton University Press 1969

[O] Ollivier, R.: Die Existenz gesschlossener Geodätischer auf kompakten Mannigfaltigkeiten. Comment. Math. Helv. 35, 146-152 (1961)

[P] Petersen, P.V.: A finiteness theorem for metric spaces. J. Differ. Geom. 31, 387395 (1990)

[P2] Petersen, P.V.: Gromov-Hausdorff convergence of metric spaces. In: Proc. Symp. Pure Math. 54, part 3, 489-504. Am. Math. Soc. Centen. Publ. 1993

[Rt] Rotman, J.: Introduction to the theory of groups. Springer 1995

[R] Rotman, R.: Upper Bounds on the Length of the Shortest Closed Geodesic on Simply Connected Manifolds. Math Z. 233, 365-398 (2000)

[T] Treibergs, A.: Estimates of volume by the length of shortest closed geodesic on a convex hypersurface. Invent. Math. 80, 481-488 (1985)

[SW] Siegel, J., Williams, F.: Variational invariants of Riemannian manifolds. Trans. Am. Math. Soc. 284, 689-705 (1984)

[Y] Yau, S.T.: Problem section in Seminar on Differential Geometry, ed. by S.T. Yau. Princeton, NJ: Princeton Univ. Press 1982 